\title{
Wilson loops and Riemann theta functions II
}

\author{
Martin Kruczenski ${ }^{a}$ and Sannah Ziama ${ }^{b}$ \\ ${ }^{a}$ Department of Physics, Purdue University, \\ 525 Northwestern Avenue, W. Lafayette, IN 47907-2036, U.S.A. \\ ${ }^{b}$ Department of Physics and Astronomy, University of Kentucky, \\ 505 Rose Street, Lexington, KY 40506-0055, U.S.A. \\ E-mail: markru@purdue.edu, spziama@pa.uky.edu
}

ABSTRACT: In this paper we extend and simplify previous results regarding the computation of Euclidean Wilson loops in the context of the AdS/CFT correspondence, or, equivalently, the problem of finding minimal area surfaces in hyperbolic space (Euclidean $\mathrm{AdS}_{3}$ ). If the Wilson loop is given by a boundary curve $\vec{X}(s)$ we define, using the integrable properties of the system, a family of curves $\vec{X}(\lambda, s)$ depending on a complex parameter $\lambda$ known as the spectral parameter. This family has remarkable properties. As a function of $\lambda, \vec{X}(\lambda, s)$ has cuts and therefore is appropriately defined on a hyperelliptic Riemann surface, namely it determines the spectral curve of the problem. Moreover, $\vec{X}(\lambda, s)$ has an essential singularity at the origin $\lambda=0$. The coefficients of the expansion of $\vec{X}(\lambda, s)$ around $\lambda=0$, when appropriately integrated along the curve give the area of the corresponding minimal area surface.

Furthermore we show that the same construction allows the computation of certain surfaces with one or more boundaries corresponding to Wilson loop correlators. We extend the area formula for that case and give some concrete examples. As the main example we consider a surface ending on two concentric circles and show how the boundary circles can be deformed by introducing extra cuts in the spectral curve.

KEYwORDS: AdS-CFT Correspondence, Long strings

ARXIV EPRINT: 1311.4950 


\section{Contents}

1 Introduction 1

2 Single curve case revisited $\quad 2$

2.1 Boundary curve 5

2.2 Analytic properties of $\mathrm{X}\left(s, p_{4}\right)$, analogy with the monodromy matrix 6

2.3 Conformal invariant ratios and Schwarzian derivative 9

$\begin{array}{lll}2.4 \text { Area } & 11\end{array}$

$\begin{array}{ll}2.5 \text { Genus } g=1 \text { case } & 13\end{array}$

3 Surfaces ending on two contours $\quad 16$

4 More than two boundary curves $\quad 19$

$\begin{array}{llr}5 & \text { Area } & 20\end{array}$

6 Conclusions $\quad 23$

A Theta functions identities $\quad 24$

$\begin{array}{ll}\text { A.1 Quasi periodicity of } \theta \text { and } \hat{\theta} & 25\end{array}$

A.2 Trisecant identity 25

A.3 Generalized cross ratio 26

$\begin{array}{lll}\text { A.4 } & \text { First derivatives } & 26\end{array}$

$\begin{array}{lll}\text { A.5 Second derivatives } & 27\end{array}$

A.6 First derivatives at $\zeta=\zeta_{s} \quad 27$

A.7 Higher derivatives at $\zeta=\zeta_{s} \quad 29$

A.8 Other identities 30

\section{Introduction}

The AdS/CFT correspondence [1-3] set on firm footing the conjectured relation between string theory and gauge theories in the planar limit. It opened the possibility of computing gauge theory quantities in the strong coupling limit by using the dual string theory. Perhaps the most important observable in gauge theories is the Wilson loop. In AdS/CFT it is related to surfaces ending on the curve associated to the Wilson loop [4-6]. In the large 't Hooft coupling limit, the surface that needs to be considered is the one of minimal area. Among all Wilson loops the simplest ones are those determined by a two dimensional, closed, smooth curve. Until recently, the only such example whose dual minimal area surface could be computed was the circle [7-11]. The minimal area surface was simply given by a half sphere. This was somewhat surprising given the known integrability properties of the system $[12,13]$. 
Recent progress in [14] provided a new, infinite parameter family of minimal area surfaces that can be used to further explore the AdS/CFT duality. In that work the minimal area surfaces were constructed analytically in terms of Riemann theta functions associated to hyperelliptic Riemman surfaces closely following previous work by M. Babich and A. Bobenko $[15,16]$. It also follows related work where Wilson loops were studied or theta functions were used in similar problems, for example in [17-29, 34-38]. ${ }^{1}$ Much of that work was motivated by the relation to scattering amplitudes $[28,29]$ which we do not pursue here. A closely related approach can also be found in [39] where integrability properties are applied to the computation of Wilson loops as minimal area surfaces. More recent developments can be found in [40-50].

Here we continue the study of such solutions and show that, in particular, they also provide an infinite parameter family of examples of surfaces ending on multiple curves. The case we concentrate on is the one of concentric curves. The main technical difference with the previous case is that a certain periodicity condition is required, which implies a restriction on the position of the cuts and on the values of the spectral parameter. Since it is only one condition, it still implies an infinite parameter family of solutions.

The paper is organized as follows: in the next section we review briefly the previous results. Since the paper is essentially a continuation of the previous one we do not attempt to make this paper self-contained and should be read in conjunction with [14]. On the other hand we give new expressions for the boundary curve and the area that were derived by simplifying the ones in [14]. After that, we present the method to find minimal area surfaces ending on multiple curves and give a few examples with plots of the corresponding surfaces and boundaries. In the following section we compute the area for the case of multiple curves in complete parallel with the result for a single curve. Finally, as illustration, we give an example of a surface ending in eight different curves and give our conclusion in the last section.

\section{Single curve case revisited}

In $[14]$ it was shown, based on $[15,16]$ how to find an infinite parameter family of minimal area surfaces ending on a curve at the boundary. The method uses integrability properties of the equations to solve them in terms of Riemann theta functions. The technique is common to many integrable non-linear differential equations and is well described in several references, for example, in the book [16].

In this section we briefly review those results and derive a simplified expression for the boundary curve which allows the explicit computation of other important quantities. For example, a simple expression is found for the Schwarzian derivative of the boundary curve. Finally, a remarkable formula for the area of the curve is found in terms of the behavior of the boundary curve near singular values of the spectral parameter.

To be concrete, we look for minimal area surfaces in hyperbolic space $\left(\mathrm{EAdS}_{3}\right)$ which can be parameterized by coordinates $(X, Y, Z>0)$ with a metric

$$
d s^{2}=\frac{d X^{2}+d Y^{2}+d Z^{2}}{Z^{2}}=\frac{d Z^{2}+d \overline{\mathrm{X}} d \mathrm{X}}{Z^{2}}, \quad \mathrm{X}=X+i Y
$$

\footnotetext{
${ }^{1}$ See e.g. [30-33].
} 
Given a contour $(X(s), Y(s), Z=0) \equiv(\mathrm{X}(s)=X(s)+i Y(s), Z=0)$ at the boundary $Z=0$ we seek a surface of minimal area ending on it and parameterized as

$$
Z(z, \bar{z}), \quad X+i Y=\mathrm{X}(z, \bar{z})
$$

where the coordinates $(z, \bar{z})$ parameterize the complex plane. In that plane we find a closed curve $z(s)$ such that $Z(z(s), \bar{z}(s))=0$. Such curve maps to the contour in the boundary of $\left(\mathrm{EAdS}_{3}\right)$ space, and the interior maps to a minimal area surface. At this time there is no generic solution to such problem of finding the minimal area surface for an arbitrary boundary. However we can give an infinite parameter family of minimal area surfaces and the corresponding contours where they end. It is an open problem if such solutions approximate all contours (and if so, in which sense).

The construction starts by choosing an auxiliary hyperelliptic Riemann surface $\mathcal{M}$, known as the spectral curve and given, as a subspace of $\mathbb{C}^{2}$ by

$$
\mu^{2}=\lambda \prod_{j=1}^{2 g}\left(\lambda-\lambda_{j}\right)
$$

where $(\mu, \lambda)$ parameterize $\mathbb{C}^{2}$ and $\lambda_{i} \neq \lambda_{j}$ if $i \neq j$. There is a reality condition that requires the set of branch points $\left\{0, \infty, \lambda_{j=1 \ldots 2 g}\right\}$ to be symmetric under the involution $T: \lambda \leftrightarrow-1 / \bar{\lambda}$. A basis of one-cycles $a_{i}, b_{i}$ is chosen such that the non-trivial intersections are $a_{i} \circ b_{j}=\delta_{i j}$ and that under the involution behave as

$$
T a_{i}=-T_{i j} a_{j}, \quad T b_{i}=T_{i j} b_{j}
$$

where $T_{i j}$ is a $g \times g$ symmetric matrix such that $T^{2}=1$.

As a matter of notation, a generic point in the Riemann surface $\mathcal{M}$ is denoted as $p_{i}=\left(\mu_{p_{i}}, \lambda_{p_{i}}\right) \in \mathcal{M}$. The coordinate $\lambda_{p_{i}}$ is called the projection of $p_{i}$ on the complex plane. The origin and infinity play an important role and are denoted as $p_{1}=0, p_{3}=\infty$. Except for the branch points, given a point $p_{4}$ in one sheet there is another point denoted as $p_{\overline{4}}$ on the other sheet of the Riemann surface such that $\lambda_{p_{4}}=\lambda_{p_{\overline{4}}}$ and $\mu_{p_{4}}=-\mu_{p_{\overline{4}}}$. The relation between $p_{4}$ and $p_{\overline{4}}$ is the hyperelliptic involution associated with $\mathcal{M}$.

Now we consider the basis of holomorphic differentials

$$
\nu_{j}=\frac{\lambda^{j-1}}{\mu(\lambda)} d \lambda, \quad i, j=1 \ldots g
$$

and define the matrices

$$
C_{i j}=\oint_{a_{i}} \nu_{j}, \quad \tilde{C}_{i j}=\oint_{b_{i}} \nu_{j}
$$

and the basis of normalized holomorphic differentials $\omega_{i=1 \cdots g}$

$$
\omega_{i}=\nu_{j}\left(C^{-1}\right)_{j i}=\sum_{j=1}^{g} C_{j i}^{-1} \lambda^{j-1} \frac{d \lambda}{\mu(\lambda)}
$$

such that

$$
\oint_{a_{i}} \omega_{j}=\delta_{i j}
$$


which defines the periodicity matrix

$$
\oint_{b_{i}} \omega_{j}=\Pi_{i j}
$$

and the theta function

$$
\theta(\zeta)=\sum_{n \in \mathbb{Z}^{g}} e^{i \pi n^{t} \Pi n+2 \pi i n^{t} \zeta}, \quad \zeta \in \mathbb{C}^{g}
$$

It also defines the Jacobi map $\phi: \mathcal{M} \rightarrow \mathbb{C}^{g}$ for which the standard notation is

$$
\phi\left(p_{4}\right)=\int_{p_{1}}^{p_{4}} \omega=\int_{1}^{4}
$$

where the point $p_{1}=0$ is chosen as a distinguished point, $p_{4}$ is an arbitrary point and $\int_{1}^{4}$ is just notation for the same function. There is an ambiguity in choosing the path of integration. Since two different paths from $p_{1}$ to $p_{4}$ can only differ by a closed cycle, $\phi\left(p_{4}\right)$ changes by

$$
\phi\left(p_{4}\right)=\int_{1}^{4} \rightarrow \int_{1}^{4}+\epsilon_{2}+\Pi \epsilon_{1}
$$

where $\epsilon_{1}$ and $\epsilon_{2}$ are vectors with integer components. Further, if $p_{4}$ is another branch point, the path $1 \rightarrow 4$ can be traced back on the lower sheet defining a generically non-trivial cycle. By choosing a certain path from $p_{1}=0$ to $p_{3}=\infty$ we define the vectors $\Delta_{1,2} \in \mathbb{Z}^{g}$ through

$$
\int_{1}^{3}=\frac{1}{2} \Delta_{2}+\frac{1}{2} \Pi \Delta_{1}
$$

The path should be chosen such that $\Delta_{1}^{t} \cdot \Delta_{2}$ is an odd integer. With this vector we define another function

$$
\hat{\theta}(\zeta)=\exp \left\{2 \pi i\left[\frac{1}{8} \Delta_{1}^{t} \Pi \Delta_{1}+\frac{1}{2} \Delta_{1}^{t} \zeta+\frac{1}{4} \Delta_{1}^{t} \Delta_{2}\right]\right\} \theta\left(\zeta+\int_{1}^{3}\right)
$$

Because $\Delta_{1}^{t} \cdot \Delta_{2}$ is odd it follows that $\hat{\theta}(-\zeta)=-\hat{\theta}(\zeta)$ and in particular $\hat{\theta}(0)=0$.

Further, consider the vector $\omega=\left(\omega_{1}, \cdots, \omega_{g}\right)$ and expand it around $p_{1}=0$. Since $p_{1}=0$ is a branch point an appropriate coordinate can be chosen as $y=-2 i \sqrt{\lambda}$, namely $\lambda=-y^{2} / 4$. It follows that

$$
\omega=\left(\omega_{1}+y^{2} \omega_{12}+y^{4} \omega_{14}+\cdots\right) d y
$$

for some constant vectors $\omega_{1}, \omega_{12}$, etc. that can be computed from eq. (2.7) by expanding $\mu(\lambda)$. Near infinity, on the other hand, an appropriate coordinate is $\tilde{y}=\frac{2}{\sqrt{\lambda}}$ and the expansion is

$$
\omega=\left(\omega_{3}+\tilde{y}^{2} \omega_{32}+\tilde{y}^{4} \omega_{34}+\cdots\right) d \tilde{y}
$$

The vectors $\omega_{1}, \omega_{3}, \omega_{12}, \omega_{32}, \ldots \in \mathbb{C}^{g}$ play an important role and it is convenient to introduce a notation for the gradient of a function along those directions

$$
\begin{aligned}
& D_{1} F(\zeta)=\left(\omega_{1} \cdot \nabla\right) F(\zeta) \\
& D_{1}^{\prime \prime} F(\zeta)=\left(\omega_{12} \cdot \nabla\right) F(\zeta)
\end{aligned}
$$




$$
\begin{aligned}
D_{3} F(\zeta) & =\left(\omega_{3} . \nabla\right) F(\zeta) \\
D_{3}^{\prime \prime} F(\zeta) & =\left(\omega_{32} \cdot \nabla\right) F(\zeta)
\end{aligned}
$$

Notice that $D_{1}^{\prime \prime}$ is a first derivative. Second derivatives are denoted e.g. as $D_{1}^{2} F(\zeta)=$ $\left(\omega_{1} \cdot \nabla\right)\left(\omega_{1} \cdot \nabla\right) F(\zeta)$. With all these ingredients in place, the solutions are given by

$$
\begin{gathered}
Z=\left|\frac{\hat{\theta}\left(2 \int_{1}^{4}\right)}{\hat{\theta}\left(\int_{1}^{4}\right) \theta\left(\int_{1}^{4}\right)}\right| \frac{|\theta(0) \theta(\zeta) \hat{\theta}(\zeta)|\left|e^{\mu z+\nu \bar{z}}\right|^{2}}{\left|\hat{\theta}\left(\zeta-\int_{1}^{4}\right)\right|^{2}+\left|\theta\left(\zeta-\int_{1}^{4}\right)\right|^{2}} \\
X+i Y=\mathrm{X}=e^{2 \mu z+2 \nu \bar{z}} \frac{\theta\left(\zeta+\int_{1}^{4}\right) \overline{\theta\left(\zeta-\int_{1}^{4}\right)}-\hat{\theta}\left(\zeta+\int_{1}^{4}\right) \overline{\hat{\theta}\left(\zeta-\int_{1}^{4}\right)}}{\left|\hat{\theta}\left(\zeta-\int_{1}^{4}\right)\right|^{2}+\left|\theta\left(\zeta-\int_{1}^{4}\right)\right|^{2}}
\end{gathered}
$$

where the vector $\zeta(z, \bar{z}) \in \mathbb{C}^{g}$ is given by

$$
\zeta=2 \omega_{1} \bar{z}+2 \omega_{3} z
$$

and the constants $\mu, \nu$ are given by

$$
\mu=-2 D_{3} \ln \theta\left(\int_{1}^{4}\right), \quad \nu=-2 D_{1} \ln \hat{\theta}\left(\int_{1}^{4}\right)
$$

which uses the directional derivatives $D_{1,3}$ defined in eq. (2.20). Notice that eq. (2.23) implies $\partial_{z} F(\zeta(z, \bar{z}))=2 D_{1} F(\zeta(z, \bar{z}))$ and $\partial_{\bar{z}} F(\zeta(z, \bar{z}))=2 D_{3} F(\zeta(z, \bar{z}))$. The solution contains an arbitrary parameter $p_{4}=\left(\mu_{p_{4}}, \lambda_{p_{4}}\right) \in \mathcal{M}$. For eq. (2.22) to define a solution it is necessary that $\left|\lambda_{p_{4}}\right|=1$. Therefore, each spectral curve $\mathcal{M}$ actually defines a one real parameter family of surfaces. It was shown in [14] that all those surfaces have the same area.

Since the boundary is at $Z=0$, to find the curve where the surface ends we need to find the zeros of $Z$ or, equivalently, the zeros of $\hat{\theta}$. Typically the zeros are given by isolated curves $z(s)$ on the world-sheet from which one can be chosen. The solution eq. (2.22) maps such curve $z(s)$ to a curve $\mathrm{X}(s)$ at the boundary $Z=0$ defining the shape of the Wilson loop. The region of the world-sheet inside the curve $z(s)$ is mapped, by eq. (2.22) to the minimal area surface ending at the curve $\mathrm{X}(s)$.

\subsection{Boundary curve}

As just described the shape of the boundary curve, or equivalently of the dual Wilson loop, follows from finding a curve $z(s)$ on the world sheet such that

$$
\hat{\theta}\left(\zeta_{s}\right)=0
$$

where

$$
\zeta_{s}=2 \omega_{1} \bar{z}(s)+2 \omega_{3} z(s)
$$

Once this function $\zeta_{s}$ is found, the shape of the curve follows from replacing $\zeta \rightarrow \zeta_{s}$ in the expression for $X$, namely eq. (2.22) to get $\mathrm{X}(s)=X(s)+i Y(s)[14]$ :

$$
\mathrm{X}(s)=e^{2 \mu z(s)+2 \nu \bar{z}(s)} \frac{\theta\left(\zeta_{s}+\int_{1}^{4}\right) \overline{\theta\left(\zeta_{s}-\int_{1}^{4}\right)}-\hat{\theta}\left(\zeta_{s}+\int_{1}^{4}\right) \overline{\hat{\theta}\left(\zeta_{s}-\int_{1}^{4}\right)}}{\left|\hat{\theta}\left(\zeta_{s}-\int_{1}^{4}\right)\right|^{2}+\left|\theta\left(\zeta_{s}-\int_{1}^{4}\right)\right|^{2}}
$$


However, it turns out that the result can be simplified by using eq. (A.9) derived in the appendix. With this identity, eq. (2.27) reduces to

$$
X(s)+i Y(s)=\mathrm{X}(s)=-e^{2 \mu z(s)+2 \nu \bar{z}(s)} \frac{\hat{\theta}\left(\zeta_{s}+\int_{1}^{4}\right)}{\hat{\theta}\left(\zeta_{s}-\int_{1}^{4}\right)}
$$

which is a much simpler expression for the boundary curve. In fact the function $\mathrm{X}(s)$ describes a family of curves parameterized by the point $p_{4}$ in the Riemann surface. Now we study the analytic properties of $\mathrm{X}\left(s, p_{4}\right)$ as a function of $p_{4}$.

\subsection{Analytic properties of $\mathrm{X}\left(s, p_{4}\right)$, analogy with the monodromy matrix}

As discussed in the previous section, the function

$$
\mathrm{X}\left(s, p_{4}\right)=-e^{2 \mu z(s)+2 \nu \bar{z}(s)} \frac{\hat{\theta}\left(\zeta_{s}+\int_{1}^{4}\right)}{\hat{\theta}\left(\zeta_{s}-\int_{1}^{4}\right)}
$$

can be thought of as a family of curves parameterized by a point $p_{4}$ on the Riemann surface $\mathcal{M}$. When $\left|\lambda_{p_{4}}\right|=1$ the curve is such that the minimal area surface can be found from eqs. (2.21) and (2.22). In this section we study the analytic properties of $\mathrm{X}\left(s, p_{4}\right)$ as a function of $p_{4}$ and show that it is well defined on the Riemann surface but not on the complex plane parameterized by $\lambda_{p_{4}}$ where it has cuts. In that manner, $\mathrm{X}\left(s, p_{4}\right)$ defines the spectral curve in the same way as the monodromy matrix does in many integrable systems. Furthermore, it turns out that $\mathrm{X}\left(s, p_{4}\right)$ has essential singularities at $p_{4}=0, \infty$, the precise behavior around the singularities determines the area of the corresponding surface. Finally, the Schwarzian derivative $\{\mathrm{X}, s\}$ is computed, and shown to have a remarkable simple dependence on $\lambda_{p_{4}}$.

To understand the properties of $\mathrm{X}\left(s, p_{4}\right)$ as a function of $p_{4}$ first it has to be proven that it is a well defined function on the Riemann surface. The reason it might not be is that there is an ambiguity in choosing the path of integration defining $\int_{1}^{4}$. Choosing another path changes the integral by a period of the theta function. Using the quasi periodicity properties of $\hat{\theta}$ i.e. eq. (A.2), it follows that

$$
\begin{aligned}
\frac{\hat{\theta}\left(\zeta_{s}+\int_{1}^{4}+\epsilon_{2}+\Pi \epsilon_{1}\right)}{\hat{\theta}\left(\zeta_{s}-\int_{1}^{4}+\epsilon_{2}+\Pi \epsilon_{1}\right)} & =e^{-4 \pi i \epsilon_{1}^{t} \zeta e^{2 \pi i\left[\Delta_{1}^{t} \epsilon_{2}-\epsilon_{1}^{t} \Delta_{2}\right]}} \frac{\hat{\theta}\left(\zeta_{s}+\int_{1}^{4}\right)}{\hat{\theta}\left(\zeta_{s}-\int_{1}^{4}\right)} \\
& =e^{-4 \pi i \epsilon_{1}^{t} \zeta} \frac{\hat{\theta}\left(\zeta_{s}+\int_{1}^{4}\right)}{\hat{\theta}\left(\zeta_{s}-\int_{1}^{4}\right)}
\end{aligned}
$$

where we used that $\Delta_{1}^{t} \epsilon_{2}-\epsilon_{1}^{t} \Delta_{2}$ is an integer since $\epsilon_{1,2}$ and $\Delta_{1,2}$ are integer vectors. Therefore the ratio of theta functions is not a well defined function on the Riemann surface. However, the exponential factor also depends on $p_{4}$ through the constants $\mu, \nu$ defined in eq. (2.24):

$$
\mu=-2 D_{3} \ln \theta\left(\int_{1}^{4}\right), \quad \nu=-2 D_{1} \ln \hat{\theta}\left(\int_{1}^{4}\right)
$$

To study their periodicity properties recall the definition

$$
D_{p_{i}} F(\zeta)=\omega\left(p_{i}\right)_{k} \nabla_{k} F(\zeta)
$$


and introduce a variable $\zeta$ to compute the derivatives

$$
\begin{aligned}
\mu \rightarrow \tilde{\mu} & =-\left.2 D_{p_{3}} \ln \theta\left(\zeta+\int_{1}^{4}+\epsilon_{2}+\Pi \epsilon_{1}\right)\right|_{\zeta=0} \\
& =-\left.2 D_{p_{3}}\left[\ln \theta\left(\zeta+\int_{1}^{4}\right)-2 \pi i \epsilon_{1}^{t} \zeta\right]\right|_{\zeta=0} \\
& =\mu+4 \pi i \epsilon_{1}^{t} \omega_{3}
\end{aligned}
$$

Similarly

$$
\nu \rightarrow \tilde{\nu}=\nu+4 \pi i \epsilon_{1}^{t} \omega_{1}
$$

Therefore the exponential factor transforms as

$$
e^{2 \mu z(s)+2 \nu \bar{z}(s)} \rightarrow e^{2 \mu z(s)+2 \nu \bar{z}(s)} e^{4 \pi i \epsilon_{1}^{t}\left(2 \omega_{3} z(s)+2 \omega_{1} \bar{z}(s)\right)}=e^{2 \mu z(s)+2 \nu \bar{z}(s)} e^{4 \pi i \epsilon_{1}^{t} \zeta_{s}}
$$

The factor precisely cancels the factor in eq. (2.31) coming from the ratio of theta functions showing that $\mathrm{X}\left(s, p_{4}\right)$ is the correct combination that is well defined on the Riemann surface.

Having proven that $\mathrm{X}\left(s, p_{4}\right)$ is well defined on the Riemann surface, it is interesting to study if it takes different values on the two sheets of the Riemann surface. This is indeed so. Changing sheets on the Riemann surface is equivalent to change $4 \rightarrow \overline{4}$ or equivalently

$$
\int_{1}^{4} \rightarrow-\int_{1}^{4}
$$

Since $\theta$ is even and $\hat{\theta}$ is odd, the functions $\mu$ and $\nu$ change sign under such change. The ratio of theta functions is obviously inverted resulting in:

$$
\mathrm{X}\left(s, p_{\overline{4}}\right)=\frac{1}{\mathrm{X}\left(s, p_{4}\right)}
$$

Therefore the function $\mathrm{X}\left(s, \lambda_{p_{4}}\right)$ defined on the complex plane has cuts. Thus, in some sense, it is analogous to the monodromy matrix. Namely, the cuts in $\mathrm{X}\left(s, \lambda_{p_{4}}\right)$ can be removed by extending the function to $\mathrm{X}\left(s, p_{4}\right)$ defined on a Riemann surface, the spectral curve of the problem.

Let us now study the analytical properties of X $\left(s, p_{4}\right)$. Riemann's theorem [54] ensures that theta functions defined on a Riemann surface have $g$ zeros and no poles. The ratio that appears in the definition of $\mathrm{X}\left(s, \lambda_{p_{4}}\right)$ therefore can have at most $g$ zeros and $g$ poles. One however is at $p_{4}=p_{1}$ and therefore cancels. In fact for the others it is clear that if $p_{4}$ is a zero then $p_{\overline{4}}$ is a pole. So $\mathrm{X}\left(s, p_{4}\right)$ has $(g-1)$ zeros and $(g-1)$ poles conjugate from each other on the Riemann surface (that means on opposite sheets). On the other hand the exponential function is quite interesting. The function

$$
\mu=-2 D_{3} \ln \theta\left(\int_{1}^{4}\right)=-2 \frac{D_{3} \theta\left(\int_{1}^{4}\right)}{\theta\left(\int_{1}^{4}\right)}
$$

has potential singularities on the $g$ zeros of $\theta\left(\int_{1}^{4}\right)$. However, the derivative also vanishes on those zeros except at $p_{4}=p_{1}$. Since $\mu$ has a pole at $p_{4}=p_{1}=0$ then $\mathrm{X}\left(s, p_{4}\right)$ has an essential singularity. The same happens at $p_{4}=p_{3}$ in view of the function $\nu$. 
To summarize: $\mathrm{X}\left(s, p_{4}\right)$ as a function of $p_{4}$ is a well defined function on the Riemann surface with $(g-1)$ zeros and $(g-1)$ poles and essential singularities at $p_{4}=p_{1}=0$ and $p_{4}=p_{3}=\infty$.

Given the symmetry $\lambda \leftrightarrow-1 / \bar{\lambda}$ it suffices to study the function near $\lambda=0$. Since $\lambda=0$ is a branch point a more appropriate coordinate $y$ can be chosen as

$$
y=-2 i \sqrt{\lambda}
$$

Using the expansion from eq. (2.15)

$$
\omega(y)=\left(\omega_{1}+\omega_{12} y^{2}+\cdots\right) d y
$$

it follows that

$$
\int_{1}^{4} \omega=\omega_{1} y+\frac{1}{3} \omega_{12} y^{3}+\cdots
$$

A simple Taylor expansion in $y$ gives

$$
\ln \left(-\frac{\hat{\theta}\left(\zeta_{s}+\int_{1}^{4}\right)}{\hat{\theta}\left(\zeta_{s}-\int_{1}^{4}\right)}\right)=1+y \frac{D_{1}^{2} \hat{\theta}\left(\zeta_{s}\right)}{D_{1} \hat{\theta}\left(\zeta_{s}\right)}+\cdots
$$

Finally the expansion

$$
\begin{aligned}
& \mu=-2 D_{13} \ln \theta(0) y+\cdots \\
& \nu=-3 \frac{2}{y}-\frac{2}{3} y\left(\frac{D_{1}^{3} \hat{\theta}(0)}{D_{1} \hat{\theta}(0)}-\frac{D_{1}^{\prime \prime} \hat{\theta}(0)}{D_{1} \hat{\theta}(0)}\right)+\cdots
\end{aligned}
$$

and eq. (A.37) in the appendix, result in

$$
\begin{aligned}
\ln \mathrm{X}= & -\frac{4}{y} \bar{z}(s) \\
& +y\left[-\frac{4}{3}\left(\frac{D_{1}^{3} \hat{\theta}(0)}{D_{1} \hat{\theta}(0)}-\frac{D_{1}^{\prime \prime} \hat{\theta}(0)}{D_{1} \hat{\theta}(0)}\right) \bar{z}(s)-4 D_{13} \ln \theta(0) z(s)+2 D_{1} \ln \theta(0)\right] \\
& +\mathcal{O}\left(y^{3}\right)
\end{aligned}
$$

This expansion is remarkable in two respects. First, the residue of the pole $\frac{1}{y}$ contains $z(s)$, namely the parameterization of the conformal world-sheet coordinate in terms of the boundary parameter. Second, as we will see later the constant term determines the area of the minimal area surface with this boundary. Actually the area of the whole family of minimal area surfaces parameterized by $p_{4}$ such that $\left|\lambda_{p_{4}}\right|=1$. It is also instructive to compute the expansion of the Schwarzian derivative $\left\{\mathrm{X}, p_{4}\right\}$ based on the expansion of $\ln \mathrm{X}$. If, as shown, $\mathrm{X}$ has an expansion

$$
\mathrm{X}=\frac{a_{1}}{y}+a_{2} y+\mathcal{O}\left(y^{3}\right)
$$

and since

$$
\{\mathrm{X}, y\}=\{\ln \mathrm{X}, y\}-\frac{1}{2}\left(\partial_{y} \ln \mathrm{X}\right)^{2}
$$


it follows that

$$
\{\mathrm{X}, y\}=-\frac{a_{1}^{2}}{2 y^{4}}+\frac{a_{1} a_{2}}{y^{2}}+\mathcal{O}(1)
$$

where the two leading terms come from the last term in eq. (2.50). It follows that

$$
\begin{aligned}
\{\mathrm{X}, y\}= & -\frac{8}{y^{4}}(\bar{z}(s))^{2} \\
& -\frac{4 \bar{z}(s)}{y^{2}}\left[-\frac{4}{3}\left(\frac{D_{1}^{3} \hat{\theta}(0)}{D_{1} \hat{\theta}(0)}-\frac{D_{1}^{\prime \prime} \hat{\theta}(0)}{D_{1} \hat{\theta}(0)}\right) \bar{z}(s)-4 D_{13} \ln \theta(0) z(s)+2 D_{1} \ln \theta(0)\right] \\
& +\mathcal{O}(1)
\end{aligned}
$$

\subsection{Conformal invariant ratios and Schwarzian derivative}

In the case of the monodromy matrix one can consider its trace which is a well defined function on the complex plane since the cuts are removed. In the present case we can consider functions of $\mathrm{X}\left(s, p_{4}\right)$ which are invariant under inversion. Those are well defined in the complex plane. For example the functions

$$
\begin{aligned}
Y^{[4]}\left(s_{1}, s_{2}, s_{3}, s_{4}, \lambda_{p_{4}}\right) & =\frac{\left(\mathrm{X}\left(s_{1}, p_{4}\right)-\mathrm{X}\left(s_{2}, p_{4}\right)\right)\left(\mathrm{X}\left(s_{3}, p_{4}\right)-\mathrm{X}\left(s_{4}, p_{4}\right)\right)}{\left(\mathrm{X}\left(s_{1}, p_{4}\right)-\mathrm{X}\left(s_{3}, p_{4}\right)\right)\left(\mathrm{X}\left(s_{4}, p_{4}\right)-\mathrm{X}\left(s_{2}, p_{4}\right)\right)} \\
Y^{[2]}\left(s_{1}, s_{2}, \lambda_{p_{4}}\right) & =\frac{\partial_{s_{1}} \mathrm{X}\left(s_{1}, p_{4}\right) \partial_{s_{2}} \mathrm{X}\left(s_{2}, p_{4}\right)}{\left(\mathrm{X}\left(s_{1}, p_{4}\right)-\mathrm{X}\left(s_{2}, p_{4}\right)\right)^{2}} \\
Y^{[1]}\left(s, \lambda_{p_{4}}\right) & =\{\mathrm{X}(s), s\}=\frac{\partial_{s}^{3} \mathrm{X}\left(s, p_{4}\right)}{\partial_{s} \mathrm{X}\left(s, p_{4}\right)}-\frac{3}{2}\left(\frac{\partial_{s}^{2} \mathrm{X}\left(s, p_{4}\right)}{\partial_{s} \mathrm{X}\left(s, p_{4}\right)}\right)^{2}
\end{aligned}
$$

are invariants depending on four, two and one point of the curve respectively. In fact they are invariant under the full Möbius group. The last one introduces the Schwarzian derivative which is conformally invariant. On the left hand side we emphasized that these are functions of $\lambda_{p_{4}}$ namely the projection of $p_{4}$ on the complex plane. As functions of $\lambda_{p_{4}}$ they have no cuts. The invariant $Y^{[4]}$ can be simply written by using eq. (2.29). On the other hand $Y^{[2]}$ requires evaluating $\partial_{s} \mathrm{X}$. From eqs. (2.29), (2.26) it follows that

$$
\partial_{s} \ln \mathrm{X}=2 \mu \partial_{s} z+2 \nu \partial_{s} \bar{z}+2 \partial_{s} z D_{1} \ln \frac{\hat{\theta}\left(\zeta_{s}+\int_{1}^{4}\right)}{\hat{\theta}\left(\zeta_{s}-\int_{1}^{4}\right)}+2 \partial_{s} \bar{z} D_{3} \ln \frac{\hat{\theta}\left(\zeta_{s}+\int_{1}^{4}\right)}{\hat{\theta}\left(\zeta_{s}-\int_{1}^{4}\right)}
$$

Using the definition of $\mu, \nu$ i.e. eq. (2.24) and eqs. (A.23), (A.29) for the derivatives of the theta function we immediately find that

$$
\partial_{s} \ln \mathrm{X}=-2 \partial_{s} z \frac{\hat{\theta}\left(2 \int_{1}^{4}\right) D_{3} \hat{\theta}(0)}{\theta^{2}\left(\int_{1}^{4}\right)} \frac{\theta^{2}(\zeta)}{\hat{\theta}\left(\zeta_{s}+\int_{1}^{4}\right) \hat{\theta}\left(\zeta_{s}-\int_{1}^{4}\right)}
$$

Equivalently

$$
\partial_{s} \mathrm{X}=2 \partial_{s} z e^{2 \mu z(s)+2 \nu \bar{z}(s)} \frac{\hat{\theta}\left(2 \int_{1}^{4}\right) D_{3} \hat{\theta}(0)}{\theta^{2}\left(\int_{1}^{4}\right)} \frac{\theta^{2}\left(\zeta_{s}\right)}{\hat{\theta}^{2}\left(\zeta_{s}-\int_{1}^{4}\right)}
$$


Thus

$$
\begin{aligned}
& Y^{[2]}\left(s_{1}, s_{2}, \lambda_{p_{4}}\right)=\left(\frac{\hat{\theta}\left(2 \int_{1}^{4}\right) D_{3} \hat{\theta}(0)}{\theta^{2}\left(\int_{1}^{4}\right)}\right)^{2} \times \\
& \quad \times \frac{4 \partial_{s} z_{1} \partial_{s} z_{2} \theta^{2}\left(\zeta_{s 1}\right) \theta^{2}\left(\zeta_{s 2}\right)}{\left[e^{\mu z_{12}+\nu \bar{z}_{12}} \hat{\theta}\left(\zeta_{s 1}+\int_{1}^{4}\right) \hat{\theta}\left(\zeta_{s 2}-\int_{1}^{4}\right)-e^{-\mu z_{12}-\nu \bar{z}_{12}} \hat{\theta}\left(\zeta_{s 1}-\int_{1}^{4}\right) \hat{\theta}\left(\zeta_{s 2}+\int_{1}^{4}\right)\right]^{2}}
\end{aligned}
$$

with the notation $z_{12}=z_{1}-z_{2}, \bar{z}_{12}=\bar{z}_{1}-\bar{z}_{2}$ and $z_{1}=z\left(s_{1}\right), z_{2}=z\left(s_{2}\right)$.

Finally, the Schwarzian derivative is harder to compute since it involves up to third order derivatives. However, it turns out that the result is remarkably simple. The second derivative can be computed in a similar way as the first one resulting in

$$
\partial_{\sigma} \ln \partial_{\sigma} \mathrm{X}=\frac{\partial_{\sigma}^{2} \mathrm{X}}{\partial_{\sigma} \mathrm{X}}=\frac{\partial_{\sigma}^{2} z}{\partial_{\sigma} z}+2 \partial_{\sigma} z\left(\mu+2 D_{3} \ln \frac{\theta(\zeta)}{\hat{\theta}\left(\zeta-\int_{1}^{4}\right)}\right)
$$

In fact now, the Schwarzian derivative can be evaluated as

$$
\begin{aligned}
\{\mathrm{X}, s\}= & \{z, s\}+8\left(\partial_{s} z\right)^{2}\left[D_{3}^{2} \ln \frac{\theta\left(\zeta_{s}\right)}{\hat{\theta}\left(\zeta_{s}-\int_{1}^{4}\right)}-\left(D_{3} \ln \frac{\theta\left(\zeta_{s}\right)}{\theta\left(\int_{1}^{4}\right) \hat{\theta}\left(\zeta_{s}-\int_{1}^{4}\right)}\right)^{2}\right] \\
& -8\left(\partial_{s} \bar{z}\right)^{2}\left(\frac{D_{1} \hat{\theta}(0) \theta\left(\int_{1}^{4}\right)}{\theta(0) \hat{\theta}\left(\int_{1}^{4}\right)}\right)^{2}
\end{aligned}
$$

The term in square brackets can be simplified by first using eq. (A.39) to replace $D_{3}^{2} \ln \hat{\theta}\left(\zeta_{s}-\right.$ $\left.\int_{1}^{4}\right)$ and then eqs. (A.20), (A.44) to further reduce the result. The second line in eq. (2.60) is simplified by the identity (A.42). In that manner the result

$$
\{\mathrm{X}, s\}=\{z, s\}-2 \lambda_{p_{4}}\left(\partial_{s} z\right)^{2}+\frac{2}{\lambda_{p_{4}}}\left(\partial_{s} \bar{z}\right)^{2}-8\left(\partial_{s} z\right)^{2}\left[\frac{D_{3}^{3} \hat{\theta}\left(\zeta_{s}\right)}{D_{3} \hat{\theta}\left(\zeta_{s}\right)}-3 \frac{D_{3}^{2} \theta\left(\zeta_{s}\right)}{\theta\left(\zeta_{s}\right)}\right]
$$

is obtained. The last term can be rewritten using eq. (A.41)

$$
\begin{aligned}
\{\mathrm{X}, s\}= & \{z, s\}-2 \lambda_{p_{4}}\left(\partial_{s} z\right)^{2}+\frac{2}{\lambda_{p_{4}}}\left(\partial_{s} \bar{z}\right)^{2} \\
& +8\left(\partial_{s} z\right)^{2}\left[2 D_{3}^{2} \ln \theta\left(\zeta_{s}\right)-\frac{D_{3}^{3} \hat{\theta}(0)}{D_{3} \hat{\theta}(0)}+\frac{D_{3}^{2} \theta(0)}{\theta(0)}\right]
\end{aligned}
$$

A completely equivalent expression can be found in terms of derivatives $D_{1}$ :

$$
\begin{aligned}
\{\mathrm{X}, s\}= & \{\bar{z}, s\}-2 \lambda_{p_{4}}\left(\partial_{s} z\right)^{2}+\frac{2}{\lambda_{p_{4}}}\left(\partial_{s} \bar{z}\right)^{2} \\
& +8\left(\partial_{s} \bar{z}\right)^{2}\left[2 D_{1}^{2} \ln \theta\left(\zeta_{s}\right)-\frac{D_{1}^{3} \hat{\theta}(0)}{D_{3} \hat{\theta}(0)}+\frac{D_{1}^{2} \theta(0)}{\theta(0)}\right]
\end{aligned}
$$

The most important point is that the dependence in $\lambda$ is very explicit in eq. (2.61). We believe this to be one of the main results of this paper since $\{\mathrm{X}, s\}$ gives a conformal invariant characterization of the Wilson loop. 


\subsection{Area}

In [14] the following formula for the finite part of the area of the minimal area surface was derived:

$$
A_{f}=16 D_{13} \ln \theta(0) \int d \sigma d \tau+\frac{1}{2} \oint \frac{\nabla^{2} \hat{\theta}\left(\zeta_{s}\right)}{\mid \nabla \hat{\theta}\left(\zeta_{s}\right)} d \ell
$$

where $(\sigma, \tau)$ are world-sheet coordinates such that $z=\sigma+i \tau$ and

$$
d \ell=\sqrt{d \sigma^{2}+d \tau^{2}}=\sqrt{\left(\partial_{s} \sigma\right)^{2}+\left(\partial_{s} \tau\right)^{2}} d s
$$

is the element of arc-length on the boundary curve $z(s)=\sigma(s)+i \tau(s)$. In this section we simplify the result and relate it to the expansion of $\mathrm{X}\left(s, p_{4}\right)$ near the singularity at $p_{4}=0$. In fact, the second term in eq. (2.66) can be rewritten as

$$
\frac{1}{2} \oint \frac{\nabla^{2} \hat{\theta}\left(\zeta_{s}\right)}{\mid \nabla \hat{\theta}\left(\zeta_{s}\right)} d \ell=\oint \partial_{z} \partial_{\bar{z}} \hat{\theta}\left(\zeta_{s}\right) \sqrt{\partial_{s} z \partial_{\sigma} \bar{z}} \partial_{z} \hat{\theta}\left(\zeta_{s}\right) \partial_{\bar{z}} \hat{\theta}\left(\zeta_{s}\right) d s
$$

Using that $\partial_{z} \hat{\theta}=2 D_{3} \hat{\theta}, \partial_{\bar{z}} \hat{\theta}=2 D_{1} \hat{\theta}$ and that the contour of integration is such that $\hat{\theta}\left(\zeta_{s}\right)=0$, the formula for the area can be rewritten as

$$
A_{f}=16 D_{13} \ln \theta(0) \int d \sigma d \theta-2 i \oint D_{1} \ln D_{3} \hat{\theta}\left(\zeta_{s}\right) d s
$$

where the sign was chosen according to the conventions in figure 1.

We can further use that

$$
\partial_{\sigma} \ln D_{3} \hat{\theta}\left(\zeta_{s}\right)=2 \partial_{s} z D_{3} \ln D_{3} \hat{\theta}\left(\zeta_{s}\right)+2 \partial_{s} \bar{z} D_{1} \ln D_{3} \hat{\theta}\left(\zeta_{s}\right)
$$

and eq. (A.35) to find

$$
A_{f}=16 D_{13} \ln \theta(0) \int d \sigma d \tau+4 i \oint D_{3} \ln \theta\left(\zeta_{s}\right) d z-\left.i \ln D_{3} \hat{\theta}\left(\zeta_{s}\right)\right|_{s_{i}} ^{s_{f}}
$$

where $s_{i, f}$ are the initial and final values of $s$. Since the curve is periodic,

$$
\left.\ln D_{3} \hat{\theta}\left(\zeta_{s}\right)\right|_{s_{i}} ^{s_{f}}=2 \pi i n
$$

for an integer $n$ known as the turning number of the curve. It is the number of times the unit normal to the curve goes around the unit circle when the point goes once around the curve. With the assumptions of figure 1 it is $n=1$. The final result for the area is therefore

$$
A_{f}=-2 \pi n+16 D_{13} \ln \theta(0) \int d \sigma d \tau+4 i \oint D_{3} \ln \theta\left(\zeta_{s}\right) d z
$$

which is simpler than the one found in the previous paper [14]. Equivalently we can write

$$
\begin{aligned}
A_{f} & =-2 \pi n-8 i D_{13} \ln \theta(0) \oint \bar{z}(s) \partial_{s} z(s) d s+4 i \oint D_{3} \ln \theta\left(\zeta_{s}\right) \partial_{s} z(s) d s \\
& =-2 \pi n+8 i D_{13} \ln \theta(0) \oint z(s) \partial_{s} \bar{z}(s) d s-4 i \oint D_{1} \ln \theta\left(\zeta_{s}\right) \partial_{s} \bar{z}(s) d s
\end{aligned}
$$




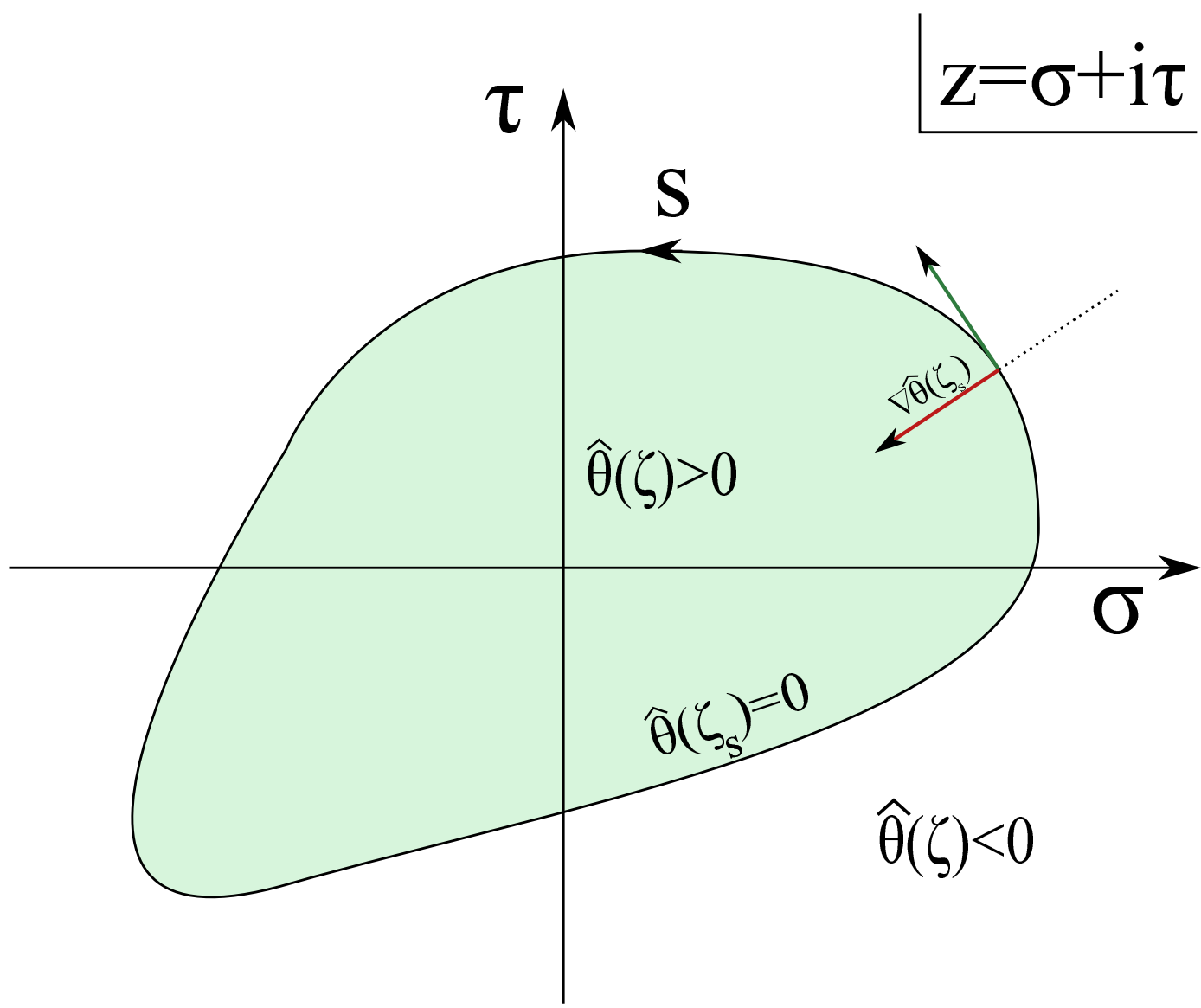

Figure 1. The world-sheet is parameterized by a complex coordinate $z$. The function $\hat{\theta}(\zeta)$ depends on $z$ through the vector $\zeta=2 \omega\left(p_{1}\right) \bar{z}+2 \omega\left(p_{3}\right) z$ where $\omega_{p_{1,3}}$ are complex vectors. Our convention is that the shaded region where $\hat{\theta}(\zeta)>0$ maps to the minimal area surface. The contour $\hat{\theta}\left(\zeta_{s}\right)=0$ maps to the boundary of the surface, i.e. the Wilson loop. The contour is parameterized counterclockwise by $s$. The gradient $\nabla \hat{\theta}\left(\zeta_{s}\right)$ is normal to the boundary and point towards the interior. Finally, the region where $\hat{\theta}\left(\zeta_{s}\right)<0$ also maps to a minimal area surface and the corresponding can be easily changed to cover that case. Slightly more involved is the case when $\hat{\theta}(\zeta)$ is purely imaginary but nothing fundamentally different changes.

Consider now the expansion in eq. (2.48). It immediately follows that

$$
A_{f}=-2 \pi n-\left.\left(\frac{2 i}{y} \oint \ln X \partial_{s} \bar{z} d s\right)\right|_{y \rightarrow 0}
$$

where we recall that $y=-2 i \sqrt{\lambda_{p_{4}}}$ is the appropriate coordinate around the branch point $p_{4}=0$. Also the integral of a total derivative was discarded. Quite remarkably, the function $\bar{z}(s)$ is also contained in the expansion, in fact

$$
-\left.\frac{1}{4} y \partial_{s} \ln \mathrm{X}\right|_{y \rightarrow 0}=\partial_{s} \bar{z}
$$


We can then write the slightly more cumbersome formula

$$
A_{f}=-2 \pi n-\frac{2 i}{\pi^{2}}\left(\oint\left[\oint_{y=0} \frac{d y}{y^{2}} \ln X(s, y)\right]\left[\oint_{y^{\prime}=0} d y^{\prime} \partial_{s} \ln X\left(s, y^{\prime}\right)\right] d s\right)
$$

The integrals on spectral parameters $y, y^{\prime}$ are complex contour integrals (given by the residues) around the points $y=0, y^{\prime}=0$. The integral along the real parameter $s$ is an integral around the boundary curve.

\subsection{Genus $g=1$ case}

In the case of genus one the theta functions reduce to Jacobi theta functions and the solutions can be written in terms of elliptic functions or, equivalently, elliptic integrals. In the previous work [14] this case was not described since it had already been studied in [25] and [26]. More recently, these solutions were analyzed including the evaluation of the first quantum correction [38]. In this subsection however, revisiting this case is useful as a starting point for the next section. For genus one there is only two cuts, namely four branch points. They are taken to be $p_{1}=0, p_{3}=\infty, \lambda_{1}=a \in \mathbb{R}, \lambda_{2}=-\frac{1}{a}$ in view of the $\lambda \leftrightarrow-1 / \bar{\lambda}$ symmetry. The spectral curve is described by the equation

$$
\mu^{2}=\lambda(\lambda-a)\left(\lambda+\frac{1}{a}\right)
$$

an is illustrated in figure 2 where it is also shown a choice of cycles $a_{1}, b_{1}$. The only holomorphic differential is

$$
\nu_{1}=\frac{d \lambda}{\mu(\lambda)}
$$

which needs to be properly normalized. Computing

$$
\begin{aligned}
& C_{1}=\oint_{a_{1}} \frac{d \lambda}{\mu(\lambda)}=2 i \int_{0}^{a} \frac{d \lambda}{\sqrt{(a-\lambda) \lambda\left(\lambda+\frac{1}{a}\right)}}=4 i \sqrt{k k^{\prime}} \mathbf{K} \\
& \tilde{C}_{1}=\oint_{b_{1}} \frac{d \lambda}{\mu(\lambda)}=-2 \int_{-\frac{1}{a}}^{0} \frac{d \lambda}{\sqrt{(a-\lambda)(-\lambda)\left(\lambda+\frac{1}{a}\right)}}=-4 \sqrt{k k^{\prime}} \mathbf{K}^{\prime}
\end{aligned}
$$

where the standard notation for elliptic functions was used (see [55] )

$$
k=\frac{a}{\sqrt{1+a^{2}}}, \quad k^{\prime}=\sqrt{1-k^{2}}=\frac{1}{\sqrt{1+a^{2}}}, \quad \mathbf{K}=K(k), \quad \mathbf{K}^{\prime}=K\left(k^{\prime}\right)
$$

The formulas from [14] reduce to

$$
\begin{aligned}
\Pi & =\tilde{C}_{1} C_{1}^{-1}=i \frac{\mathbf{K}^{\prime}}{\mathbf{K}} \\
\omega & =-\frac{i}{4 \sqrt{k k^{\prime}} \mathbf{K}} \frac{d \lambda}{\mu(\lambda)} \\
\omega_{1} & =-\omega_{3}=\frac{i}{4 \sqrt{k k^{\prime}} \mathbf{K}}
\end{aligned}
$$


We also have $\Delta_{1}=1, \Delta_{2}=1$. Taking the involution $T=-1$ we find

$$
\bar{\Pi}=-T \Pi T, \quad T \Delta_{1,2}=-\Delta_{1,2} \quad T \omega_{3}=\bar{\omega}_{1}
$$

as it should be for the reality condition to be satisfied. Let us introduce also the half-period $\hat{a}$ that defines $\hat{\theta}$ and which is a zero of $\theta$ :

$$
\hat{a}=\frac{1+\Pi}{2}
$$

Now it is possible to define the variable $\zeta$

$$
\zeta=2 \omega_{1} \bar{z}+2 \omega_{3} z=\frac{\tau}{\sqrt{k k^{\prime}} \mathbf{K}}
$$

where $z=\sigma+i \tau$. Now we can relate the Riemann theta functions to Jacobi Theta functions (we use the convention in [55]) by:

$$
\theta(\zeta)=\theta_{3}(\pi \zeta, q), \quad \hat{\theta}(\zeta)=\theta\left[\begin{array}{c}
\Delta_{1} \\
\Delta_{2}
\end{array}\right](\zeta)=-\theta_{1}(\pi \zeta, q), \quad \text { with } \quad q=e^{i \pi \Pi},
$$

Some convenient formulas are [55]

$$
D_{1} \theta(\hat{a})=\omega_{1} \theta_{3}^{\prime}\left(\frac{1+\Pi}{2}\right)=i \pi \omega_{1} q^{-\frac{1}{4}} \theta^{\prime}(0)
$$

and

$$
\theta_{3}(0)=\sqrt{\frac{2 \mathbf{K}}{\pi}}, \quad \theta_{1}^{\prime}(0)=\theta_{2}(0) \theta_{3}(0) \theta_{4}(0) \quad \theta_{2}(0) \theta_{4}(0)=\sqrt{k k^{\prime}} \theta_{3}^{2}(0)
$$

The world-sheet metric (or Lagrange multiplier $\Lambda$ ) is determined by

$$
e^{2 \alpha}=\frac{\theta_{3}^{2}(\pi \zeta)}{\theta_{1}^{2}(\pi \zeta)}=\frac{1}{k k^{\prime}}\left(\frac{\operatorname{dn}\left(\frac{2 \tau}{\sqrt{k k^{\prime}}}, k\right)}{\operatorname{sn}\left(\frac{2 \tau}{\sqrt{k k^{\prime}}}, k\right)}\right)^{2}
$$

In the last equation $\alpha$ was written in terms of Jacobi elliptic functions, perhaps the easiest expression to check the validity of the cosh-Gordon equation

$$
\frac{1}{4} \partial_{\tau}^{2} \alpha=e^{2 \alpha}+e^{-2 \alpha}
$$

for such function. The last step is to choose the spectral parameter $\lambda$ such that $|\lambda|=1$. In terms of $\lambda$ we define

$$
v=\int_{1}^{4}=-\frac{i}{4 \sqrt{k k^{\prime}} \mathbf{K}} \int_{0}^{\lambda} \frac{d \lambda}{\mu(\lambda)}
$$

The solution can then be written in terms of Jacobi theta functions as

$$
\begin{aligned}
\mu & =2 \pi \omega_{1} \partial_{v} \ln \theta_{3}(v) \\
\nu & =\mp 2 \pi \omega_{1} \partial_{v} \ln \theta_{1}(v) \\
\psi_{1} & =-2 \pi \omega_{1} \frac{\theta_{1}^{\prime}(0) \theta_{1}(v) \theta_{1}(x+v)}{\theta_{3}(0) \theta_{3}(v) \theta_{1}(x)} e^{-\frac{\alpha}{2}} e^{\mu z+\nu \bar{z}}
\end{aligned}
$$




$$
\begin{aligned}
\psi_{2} & = \pm \frac{\theta_{3}(x+v)}{\theta_{3}(x)} e^{\frac{\alpha}{2}} e^{\mu z+\nu \bar{z}} \\
\tilde{\psi}_{1} & =2 \pi \omega_{1} \frac{\theta_{1}^{\prime}(0) \theta_{1}(v) \theta_{1}(x-v)}{\theta_{3}(0) \theta_{3}(v) \theta_{1}(x)} e^{-\frac{\alpha}{2}} e^{-\mu z-\nu \bar{z}} \\
\tilde{\psi}_{2} & = \pm \frac{\theta_{3}(x-v)}{\theta_{3}(x)} e^{\frac{\alpha}{2}} e^{-\mu z-\nu \bar{z}} \\
\psi_{1} \tilde{\psi}_{2}-\psi_{2} \tilde{\psi}_{1} & =\left|\frac{\theta_{1}(2 v) \theta_{3}(0)}{\theta_{3}^{2}(v)}\right| \\
\zeta & =2 \omega_{1}( \pm \bar{z}-z) \\
\lambda & =\left(\frac{\theta_{1}(v)}{\theta_{3}(v)}\right)^{2}
\end{aligned}
$$

where $v$ is an additional parameter which corresponds to $\int_{1}^{4}$ in eq. (2.22). The formulas for $\psi_{1,2}$ and $\tilde{\psi}_{1,2}$ refer to the functions defined in [14] and are included for completeness. They are not used in this paper. Finally, the solution is written as

$$
\begin{aligned}
Z & =\left|\frac{\theta_{1}(2 v) \theta_{3}(0)}{\theta_{3}^{2}(v)} e^{-2 \mu z-2 \nu \bar{z}}\right| \frac{\theta_{1}(x) \theta_{3}(x)}{\left|\theta_{1}(x+v)\right|^{2}+\left|\theta_{3}(x+v)\right|^{2}} \\
X+i Y & =e^{-2 \bar{\mu} \bar{z}-2 \bar{\nu} z} \frac{\theta_{3}(x+v) \bar{\theta}_{3}(x-v)-\theta_{1}(x+v) \bar{\theta}_{1}(x-v)}{\left|\theta_{1}(x+v)\right|^{2}+\left|\theta_{3}(x+v)\right|^{2}}
\end{aligned}
$$

As before the boundary curves are found by finding the zeros of $\hat{\theta}=-\theta_{1}$. Since the variable $\zeta_{+}$depends only on $\tau$ ( or $\zeta_{-}$on $\sigma$ ), the world-sheet region is a strip between two zeros of $\theta_{1}$. Generically the edges of the strip map to two infinite boundary curves with the shape of a spiral. However if we take $\lambda=1$ for $a>1$ and $\lambda=-1$ for $a<1$ we have the following interesting result

- $a>1, \lambda=1$, the Wilson loop is given by two concentric circles of radii $r_{1,2}$, satisfying

$$
\ln \frac{r_{2}}{r_{1}}=\pi \operatorname{Re}\left(\frac{\theta_{3}^{\prime}(v)}{\theta_{3}(v)}+\frac{\theta_{1}^{\prime}(v)}{\theta_{1}(v)}\right)
$$

The ratio, by definition, is smaller than one, but, analyzing the previous result, it shows that it has a minimum value larger than zero. For each value of the ratio $\frac{r_{2}}{r_{1}}$ in that range there are two possible values of $v$. However they do not give the same solution, namely, for each allowed ratio $\frac{r_{2}}{r_{1}}$ there are two solutions, one world-sheet is close to the boundary and the other extends towards the interior.

- $a<1, \lambda=-1$ the Wilson loop is given by a cusp.

The following limits are of interest

- $a \rightarrow \infty$ gives a circle.

- $a \rightarrow 1^{+}$gives two parallel lines.

- $a \rightarrow 1^{-}$gives two parallel lines. 


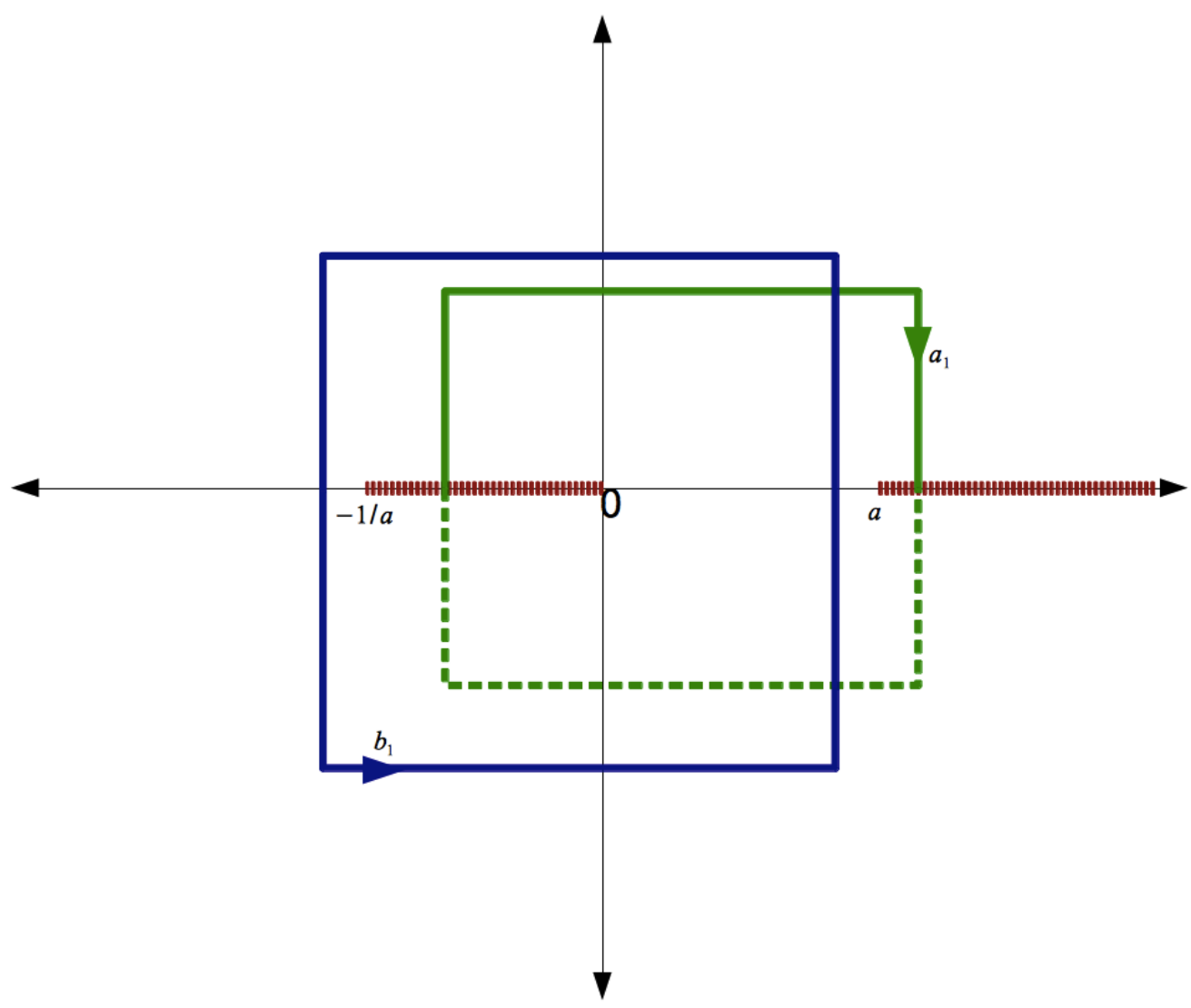

Figure 2. g=1 Riemann surface.

- $a \rightarrow 0$ gives a straight line.

For the case $a=1$ corresponding to two parallel line the spectral curve coincides with the one given in [39]:

$$
\mu^{2}=\lambda\left(\lambda^{2}-1\right)
$$

suggesting that both approaches are equivalent.

\section{Surfaces ending on two contours}

In the previous section we discussed, following [26] a solution ending on two concentric circles and corresponding to two cuts. It seems evident that adding two small cuts to the spectral curve will just slightly deform the solution. This is correct except that generically the periodicity is broken and therefore the curves become infinite spirals instead of two closed curves. To be precise, the theta functions are periodic but there is also the exponential factor in eq. (2.28) which is periodic when the exponent is purely imaginary, a condition that needs to be satisfied. Both periods have to match. It is clear that for particular positions of the cuts the periodicity would be restored but it requires some numerical effort to find them. In this section we present several examples. The number of 


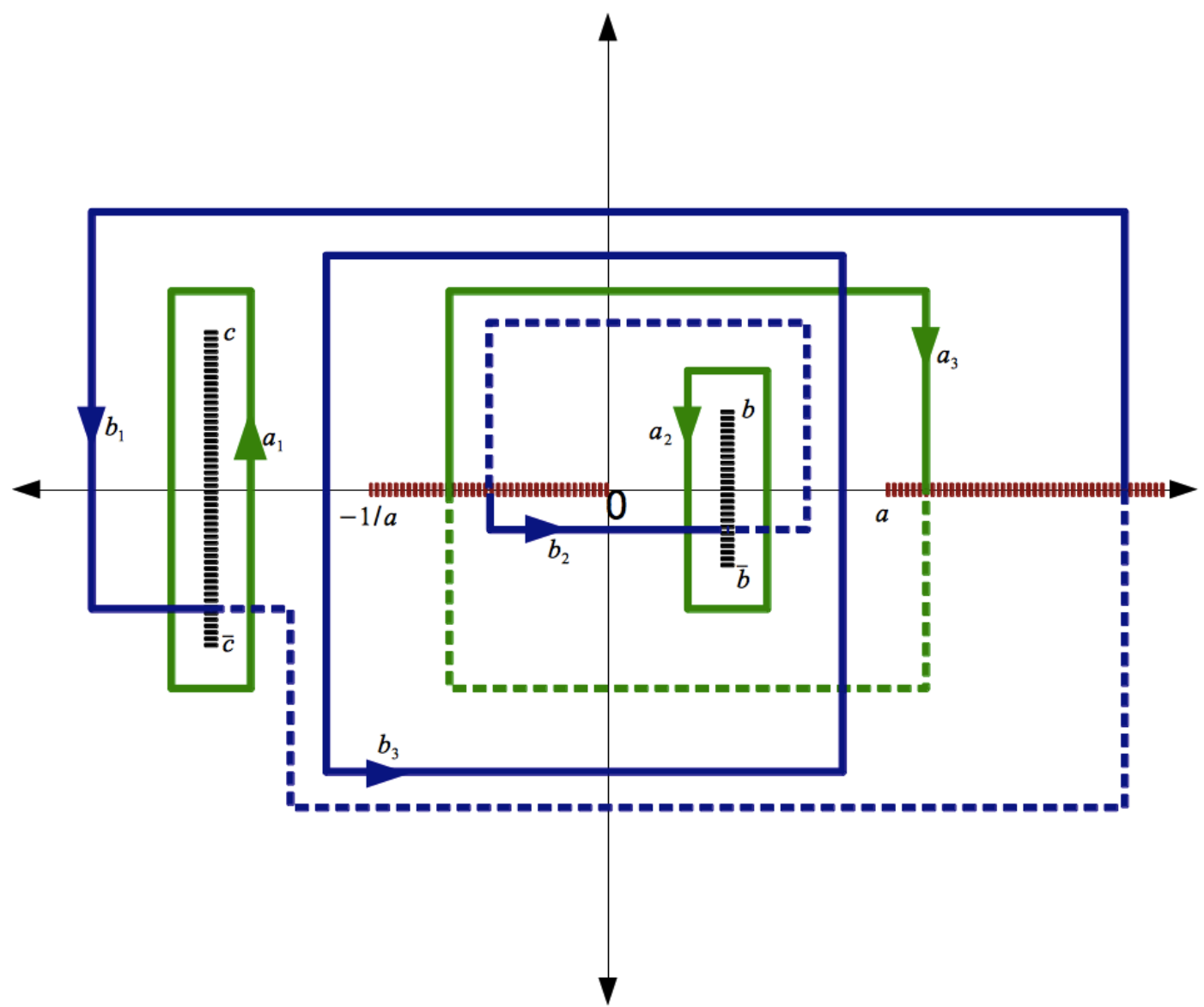

Figure 3. $\mathrm{g}=3$ hyperelliptic Riemann surface.

branch cuts is four, the situation is depicted in figure 3 where also the choice of cycles is made evident. Due to the involution $\lambda \rightarrow-1 / \bar{\lambda}$, the branch point $c=-1 / b$. The points $\bar{b}$ and $\bar{c}$ are the complex conjugates of $b$ and $c$, respectively.

As we shrink the $b \bar{b}$ and $c \bar{c}$ branch cuts we should get results close to the concentric circles for $a>1$. Unlike the $g=1$ case, even if we take $\lambda=1$, the solutions are not automatically periodic; we must pick a suitable value of $a>1$ as we move $b$ to get periodic concentric curves.

The ratio of Riemann theta functions in eq. (2.28) is periodic if the argument shifts by an integer vector, also, the exponential function is periodic under a $2 \pi$ shift in the imaginary direction. Both periods have to match. This can be achieved in practice by moving the branch points around. We found values for $a$ and $b$ such that we can match $2,3,4$ and 5 periods of the theta function into a single period of the exponential. In each case we obtained concentric Wilson loops. The curves deviate from a circle in a way such that the periodicity is manifest as can be seen in figures 4 and 5. The corresponding positions of the branch points is shown in table 1 below. It is also possible to fit one period of the theta function into e.g. two periods of the exponential in (2.28). It turns out that we get a boundary made of self-intersecting concentric curves. For appropriate choices of $a$ and $b$ 


\begin{tabular}{|l|l|l|l|l|}
\hline & $\mathrm{n}=2$ & $\mathrm{n}=3$ & $\mathrm{n}=4$ & $\mathrm{n}=5$ \\
\hline & $a=1.28088$ & $a=1.102149$ & $a=1.035312$ & $a=1.0304752$ \\
\hline & $b=0.5+0.01 i$ & $b=0.5+0.1 i$ & $b=0.7+0.2 i$ & $b=0.7+0.3 i$ \\
\hline
\end{tabular}

Table 1. Positions of the branch points; $\mathrm{n}$ corresponds to the number of periods of the exponential prefactor in each period of the ratio of theta function.
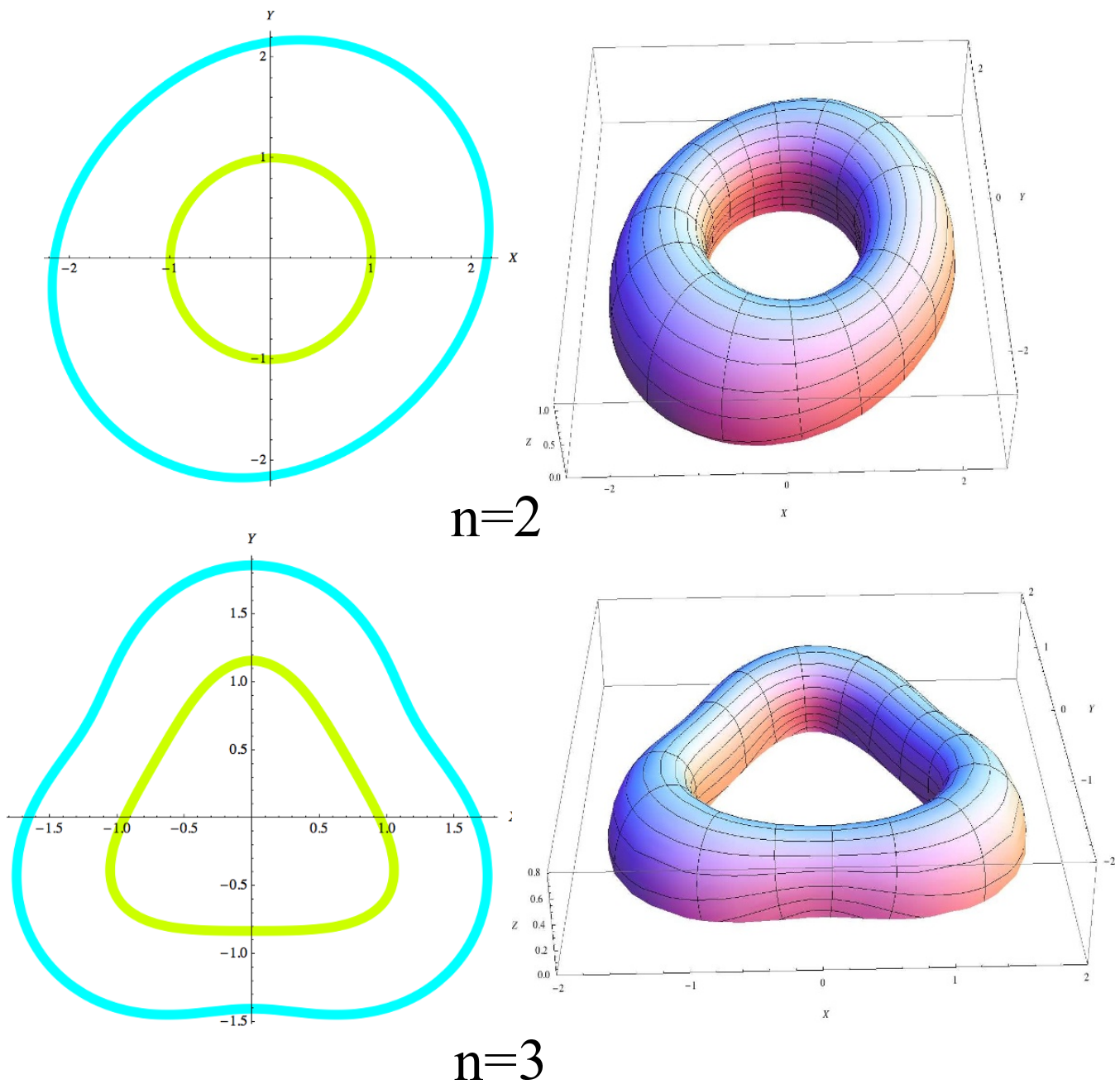

Figure 4. Wilson loops and corresponding minimal area surfaces for two concentric curves. In this case, two and three periods of the theta functions are fit into one period of the exponential as is evident from the shape of the curves.

each individual curve resembles a circle which is described twice as shown in figure 6 . It is also made clear the claim that as we shrink the $b \bar{b}$ branch cut the surface approaches the one corresponding to the concentric circular Wilson loops. 

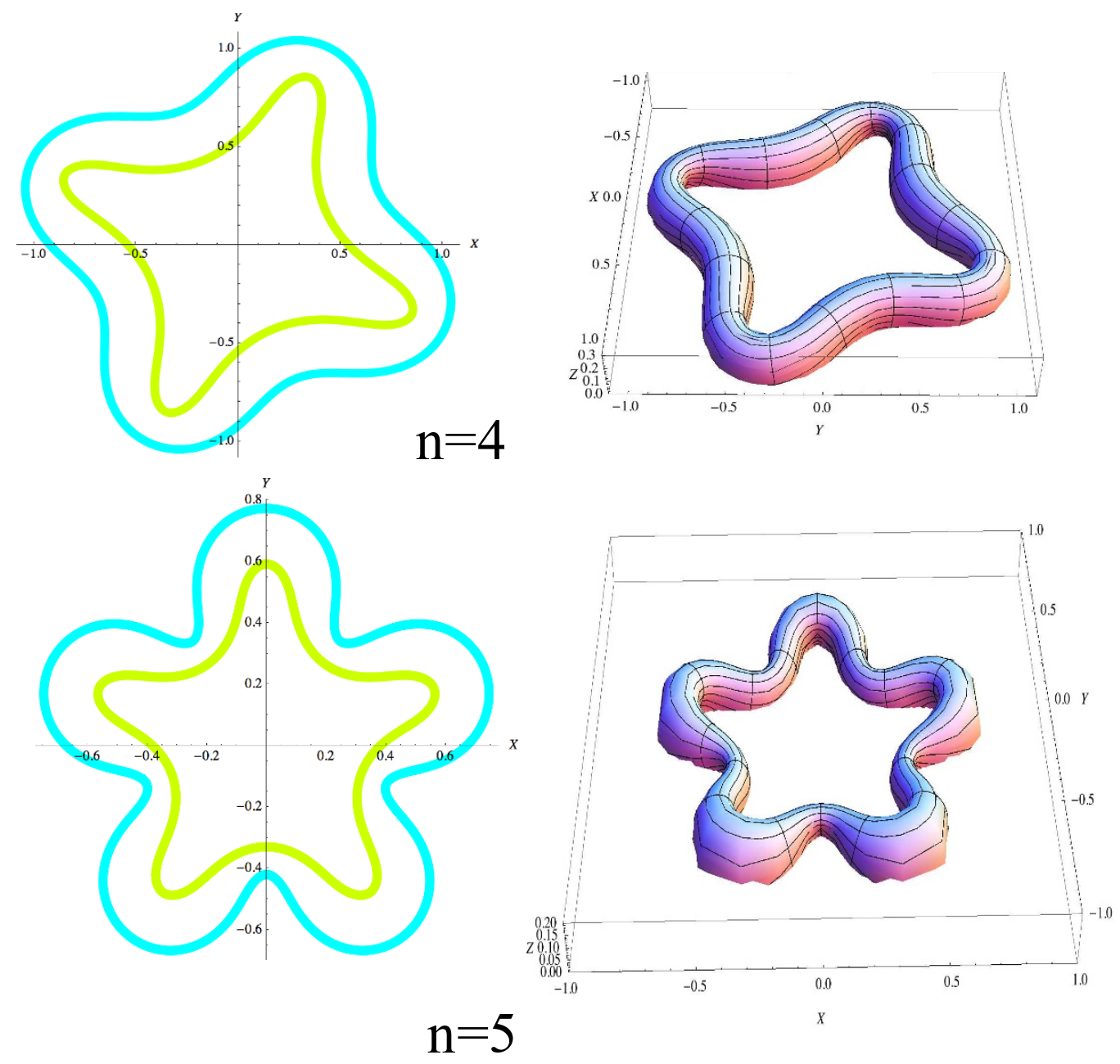

Figure 5. Wilson loops and corresponding minimal area surfaces for two concentric curves. Now, four and five periods of the theta functions are fit into one period of the exponential as is evident from the shape of the curves.

\section{More than two boundary curves}

As the size of the cuts is varied, closed curves appear in the region between the two periodic curves giving rise to surfaces with more boundaries. An example is shown in figure 7 . The curves depicted are zeros of $\hat{\theta}$ in the world-sheet and correspondingly of the function $Z(z, \bar{z})$. That means that they map into multiple curves at the boundary. The region surrounded by one of the closed curves maps into a minimal area surface ending in the corresponding single curve. On the other hand the complement of those regions maps to a minimal area surface ending in multiple curves, those closed curves we described plus two infinite curves (of spiral shape) given by the upper and lower periodic curves. Again, for $\lambda=1$ and particular positions of the cuts, the period of those curves match those of the exponential and the result is a surface ending in multiple closed curves. A particular example is illustrated in figures 8,9 and 10. Admittedly, the surface is not simple to describe or plot since it ends in eight different and self-intersecting curves. The boundary curves are shown 

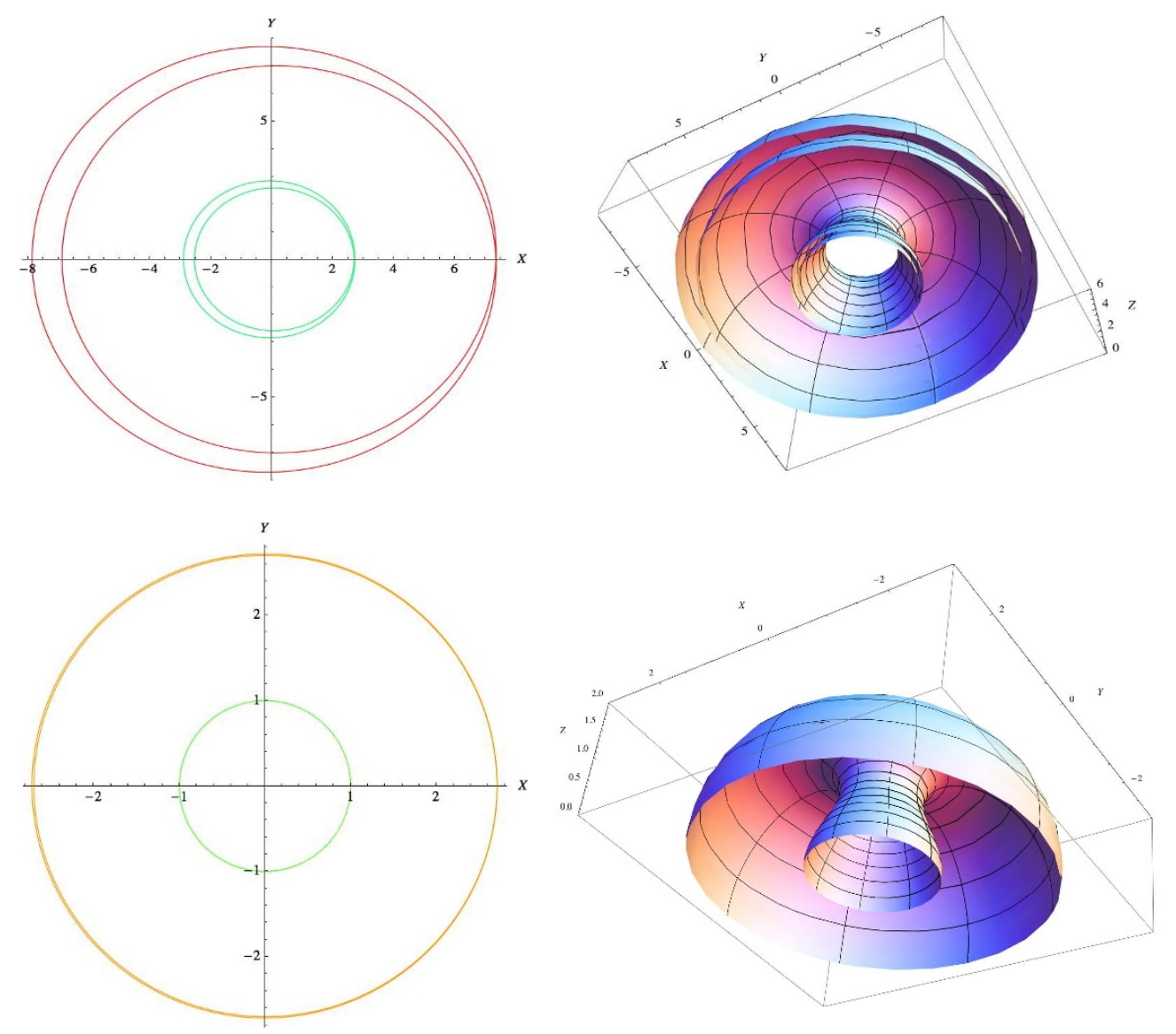

Figure 6. In this case, two periods of the exponential are fit in two periods of the theta function giving rise to concentric self-intersecting curves. In the first example the position of the cuts is given by $a=2.412712, b=2+0.05 I$ and in the second $a=2.414205, b=2+0.003 I$. The second one is shown as illustration of the fact that the surface can be made to approximate the (double cover) of the concentric circles.

in figure 9, notice however that different scales are used. As an intermediate representation, contours of equal $Z$ are depicted in figure 8. Finally, the surface is plotted in figure 10.

\section{Area}

Consider again the surfaces ending on two concentric curves, namely those in figure 4,5 and compute their area. The formula in eq. (2.75) applies to this case if we take the turning number of the curve to be $n=0$. Remember that this is the turning number of the boundary curve in the world-sheet. In figure 7 it is clear that, as one moves along the upper and lower curves, the tangent to the curves does not wrap around the unit circle. The area of the world-sheet can also be explicitly written term of a parameterization $\tau_{1,2}(\sigma)$ of the 


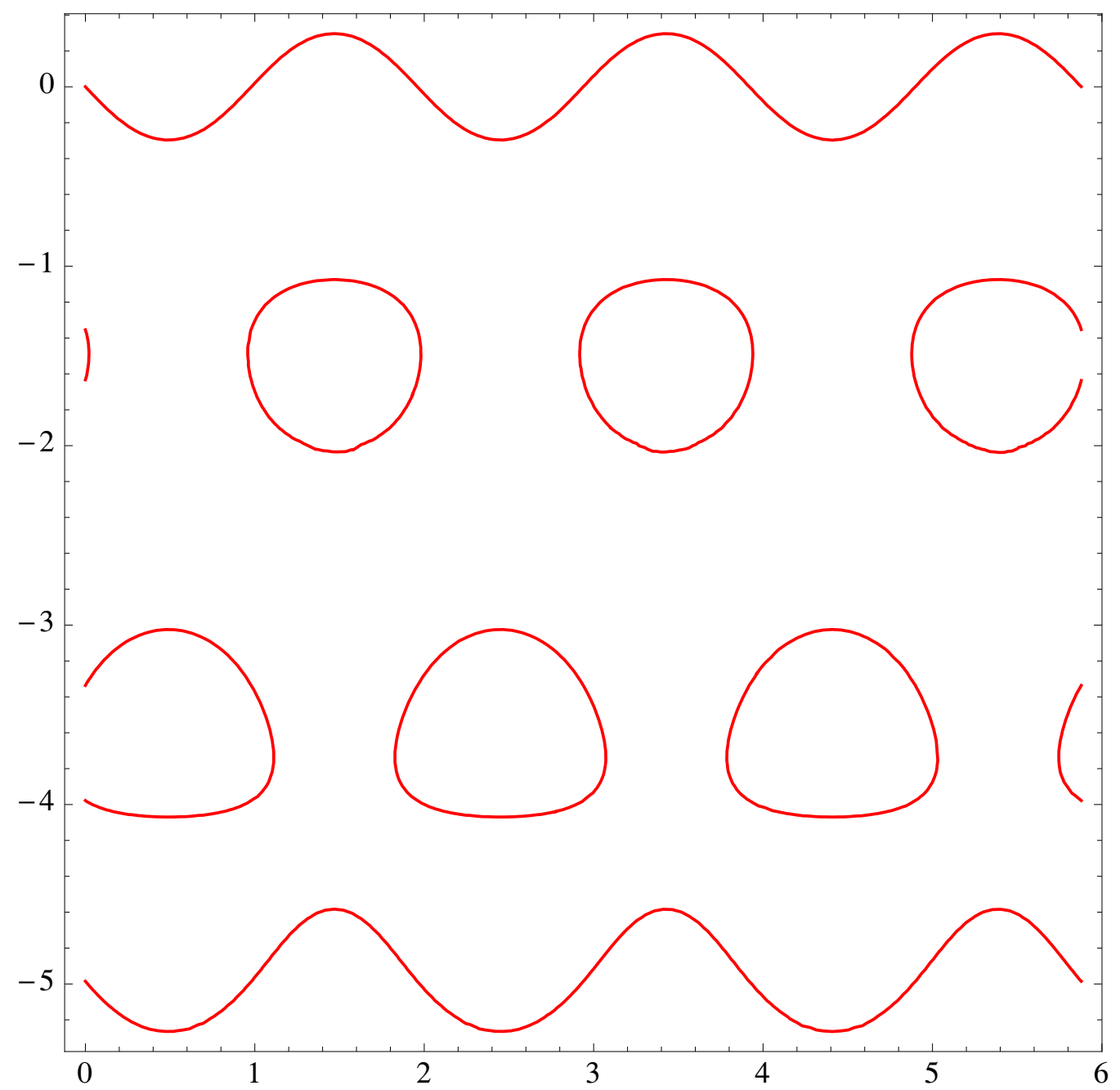

Figure 7. World-sheet of the string where the zeros of $\hat{\theta}$ are depicted. Those curves map to the boundary. For particular position of the cuts, the period of the figure in the $\sigma$ direction matches the period of the exponential function and a surface ending in several closed curves is obtained.

upper and lower curves giving

$$
\begin{aligned}
A_{f} & =4 D_{p_{1} p_{3}} \log \theta(0) \int\left(\tau_{2}(\sigma)-\tau_{1}(\sigma)\right) d \sigma+\frac{1}{2}\left(\int_{\tau_{2}(\sigma)}+\int_{\tau_{1}(\sigma)}\right) \frac{\nabla^{2} \hat{\theta}}{|\nabla \theta|} d l \\
& =-2 \Im\left\{D_{13} \log \theta(0) \oint z d \bar{z}+\left(\int_{\tau_{2}(\sigma)}-\int_{\tau_{1}(\sigma)}\right) D_{1} \log \theta(\zeta) d \bar{z}\right\}
\end{aligned}
$$

Recall also that the total area is given by

$$
A_{\text {total }}=\frac{L}{\epsilon}+A_{f}
$$

where $L$ is the length of the Wilson loop and $\epsilon \rightarrow 0$ is the regulator defined by cutting the surface at $Z=\epsilon$. The analytical results thus obtained are in agreement with a direct numerical evaluation of the Area as shown in table 2. 


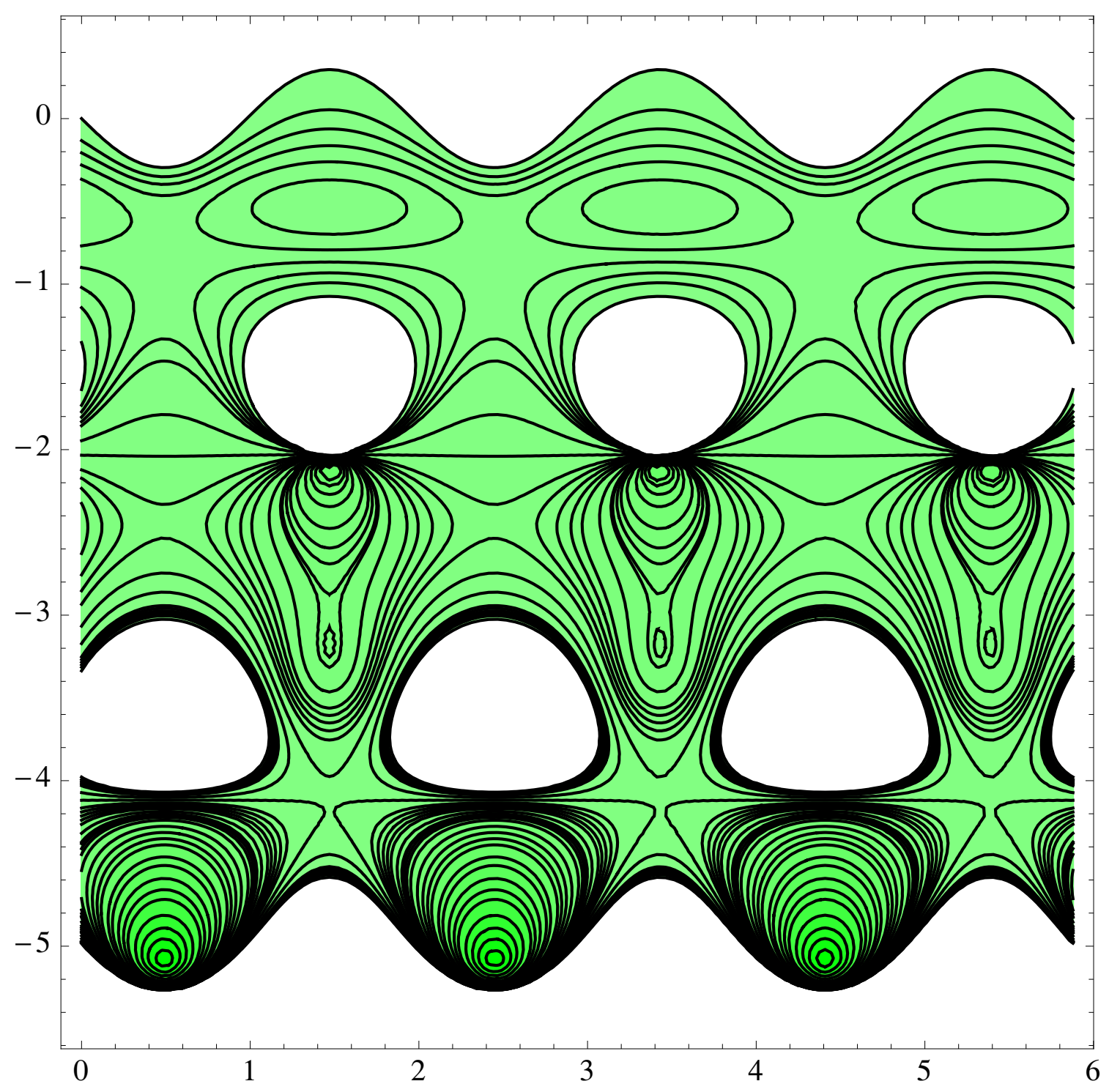

Figure 8. Contour lines indicating the surface heights above the boundary Wilson loop.

\begin{tabular}{|c|c|c|c|c|}
\hline Areas & $\mathrm{n}=2$ & $\mathrm{n}=3$ & $\mathrm{n}=4$ & $\mathrm{n}=5$ \\
\hline$A_{\text {total }}$ by fit & $-13.80+\frac{19.8644}{\epsilon}$ & $-20.55+\frac{17.25}{\epsilon}$ & $-40.23+\frac{12.23}{\epsilon}$ & $-55.48+\frac{9.64}{\epsilon}$ \\
\hline$A_{\text {total }}$ by eq. (5.2) & $-13.80+\frac{19.86}{\epsilon}$ & $-20.55+\frac{17.25}{\epsilon}$ & $-40.27+\frac{12.23}{\epsilon}$ & $-55.66+\frac{9.63658}{\epsilon}$ \\
\hline$A_{f}$ by eq. (2.74) & -13.80 & -20.55 & -40.27 & -55.66 \\
\hline
\end{tabular}

Table 2. Numerical values of the Area of minimal surfaces in figures 4 and 5 . 

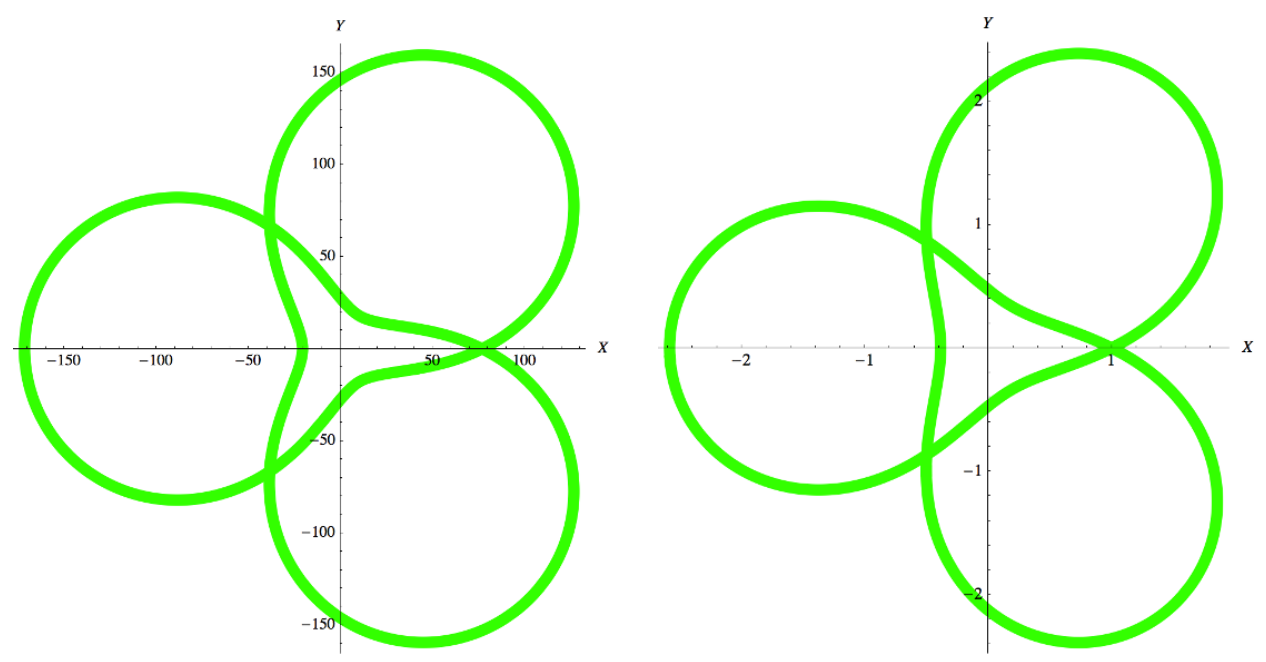

Figure 9. Image on the boundary of the curves depicted in figure 7 .

\section{Conclusions}

In this paper we have simplified and extended the results in [14]. Given a boundary curve $\mathrm{X}(s)$, a one complex parameter family of curves $\mathrm{X}(s, \lambda)$ can be given by solving the linear problem associated with the flat connection. Studying the analytical properties of $\mathrm{X}(s, \lambda)$ it turns put that the function is naturally defined on a hyperelliptic Riemann surface $\mathcal{M}$, the spectral curve. We then denote it as $\mathrm{X}\left(s, p_{4}\right)$ with $p_{4} \in \mathcal{M}$. In that way it plays a similar role to the monodromy matrix for standard integrable systems. The first part of the paper is devoted to the study of such function. In particular we find a simple expression for the Schwarzian derivative $\{\mathrm{X}, s\}$. It is also shown that $\mathrm{X}\left(s, p_{4}\right)$ has an essential singularity at $p_{4} \rightarrow 0$ and the behavior near the singularity determines the area of the surface.

The second part of the paper is devoted to the study of surfaces ending in multiple contours. In particular examples for surfaces ending in two or more contours are given, generalizing previous results of Drukker and Fiol [26]. Again, a relatively simple expression is given for the area of such curves.

\section{Acknowledgments}

The motivation for pursuing this research is the result of several discussions with $\mathrm{N}$. Drukker, J. Maldacena, A. Sever, A. Tseytlin and P. Vieira. In addition, M.K. is grateful to V. Enolskii and the other participants of the Algebro-geometric Methods in Fundamental Physics, 515 WE-Heraeus Seminar for helpful insights into the mathematical aspects of this work.

The authors want to thank the Simons Center for Geometry and Physics and M.K. to Perimeter Institute for hospitality while part of this work was being done.

The work of M.K. was supported in part by NSF through a CAREER Award PHY0952630 and by DOE through grant DE-SC0007884 and that of S.Z. in part by the Lyman T. Johnson Postdoctoral Fellowship. 


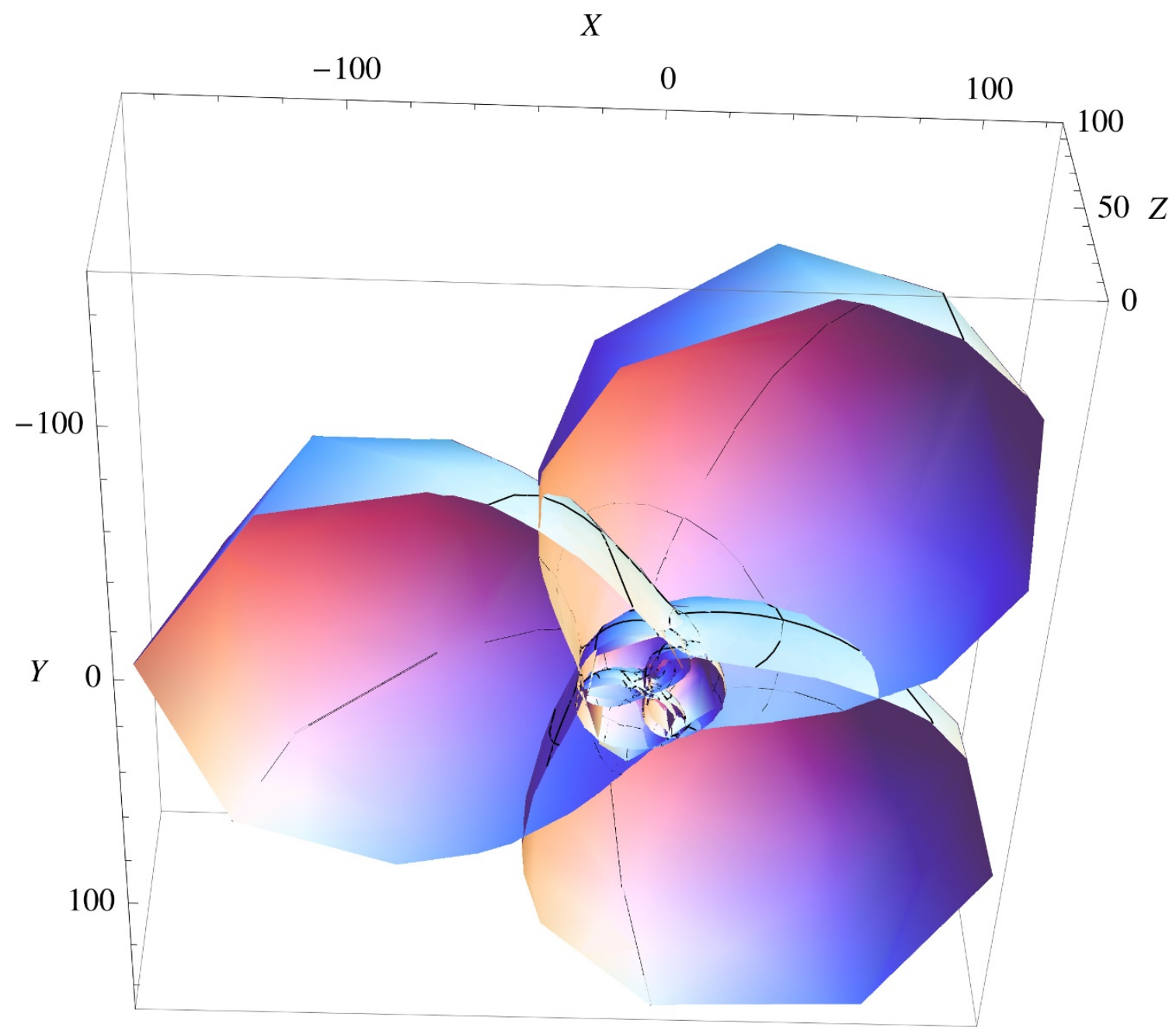

Figure 10. Example of minimal area surface embedded in Euclidean $A d S_{3}$ and ending on multiple contours.

\section{A Theta functions identities}

In this appendix we derive several identities for the Riemann theta functions that are needed in the main text. Some of the calculations are done in detail to illustrate the procedure. We use a basic knowledge of the theory of theta functions as can be found, for example, in the classic book on Riemann surfaces by Farkas and Kra [54]. In fact everything follows from the quasi periodicity property and the trisecant identity. Specialized books on theta functions such as [51-53] contain many of these results, we just briefly rederive them in this particular context and using the notation of this paper. 


\section{A.1 Quasi periodicity of $\theta$ and $\hat{\theta}$}

Given two integer vectors $\epsilon_{1,2}$ it follows from the definition of $\theta(\zeta)$ and $\hat{\theta}(\zeta)$, i.e. eqs. (2.10), (2.14) that

$$
\theta\left(\zeta+\epsilon_{2}+\Pi \epsilon_{1}\right)=e^{-2 \pi i\left[\epsilon_{1}^{t} \zeta+\frac{1}{2} \epsilon_{1}^{t} \Pi \epsilon_{1}\right]} \theta(\zeta)
$$

and

$$
\hat{\theta}\left(\zeta+\epsilon_{2}+\Pi \epsilon_{1}\right)=e^{-2 \pi i\left[\epsilon_{1}^{t} \zeta+\frac{1}{2} \epsilon_{1}^{t} \Pi \epsilon_{1}\right]} e^{i \pi\left[\Delta_{1}^{t} \epsilon_{2}-\epsilon_{1}^{t} \Delta_{2}\right]} \hat{\theta}(\zeta)
$$

As discussed in eq. (2.12), the function $\phi\left(p_{4}\right)=\int_{1}^{4}$ is holomorphic on $\mathcal{M}$ but defined only up to integer periods. The quasi periodicity of the theta function allows to write holomorphic functions on $\mathcal{M}$ as ratios

$$
f\left(p_{4}\right)=\frac{\prod_{v=1}^{n} \theta\left(\zeta_{v}+\int_{1}^{4}\right)}{\prod_{\tilde{v}=1}^{n} \theta\left(\tilde{\zeta}_{\tilde{v}}+\int_{1}^{4}\right)}, \quad \text { with } \quad \sum_{v=1}^{n} \zeta_{v}=\sum_{\tilde{v}=1}^{n} \tilde{\zeta}_{\tilde{v}}
$$

for arbitrary constant vectors $\zeta_{v=1 \cdots n}, \tilde{\zeta}_{\tilde{v}=1 \cdots n}$. Indeed, under such condition $f\left(p_{4}\right)$ is independent of the path chosen in $\int_{1}^{4}$ and therefore is a well defined function on the Riemann surface. A particular useful example is the generalized cross ratio defined in eq. (A.5) below.

\section{A.2 Trisecant identity}

By studying the analytic properties of ratios such as those in eq. (A.2) Fay derived the following fundamental identity known as trisecant identity [54]:

$$
\theta(\zeta) \theta\left(\zeta+\int_{j}^{i}+\int_{k}^{l}\right)=\gamma_{i j k l} \theta\left(\zeta+\int_{j}^{i}\right) \theta\left(\zeta+\int_{k}^{l}\right)+\gamma_{i k j l} \theta\left(\zeta+\int_{k}^{i}\right) \theta\left(\zeta+\int_{j}^{l}\right)
$$

valid for an arbitrary vector $\zeta \in \mathbb{C}$ and four arbitrary points $p_{i, j, k, l}$ on the Riemann surface. The coefficient $\gamma_{i j k l}$ is called a generalized crossed ratio and defined as

$$
\gamma_{i j k l}=\frac{\theta\left(a+\int_{k}^{i}\right) \theta\left(a+\int_{l}^{j}\right)}{\theta\left(a+\int_{l}^{i}\right) \theta\left(a+\int_{k}^{j}\right)}
$$

where, $a$ is a non-singular zero of the theta function i.e. $\theta(a)=0$ but $|\nabla \theta| \neq 0$. Under such conditions it is an important property of the cross ratio that it does not depend on the zero $a$ chosen to defined it. In this paper we defined $\hat{\theta}$, see eq. (2.14), through the odd period $\int_{1}^{3}$. Since $\hat{\theta}(0)=0$ it implies that $\theta\left(\int_{1}^{3}\right)=0$. Setting $a=\int_{1}^{3}$ in the cross ration gives

$$
\gamma_{i j k l}=\frac{\hat{\theta}\left(\int_{k}^{i}\right) \hat{\theta}\left(\int_{l}^{j}\right)}{\hat{\theta}\left(\int_{l}^{i}\right) \hat{\theta}\left(\int_{k}^{j}\right)}
$$

The trisecant identity plays a central role in using the theta function to solve non-linear differential equations in integrable systems. For us, it is the starting point for deriving all the identities below. It is interesting to note that in string theory it has an interpretation as a bosonization identity [56]. 


\section{A.3 Generalized cross ratio}

As it was just stated, $\gamma_{i j k l}$ in eq. (A.5) is independent of $a$ as long as $a$ is a zero of the theta function $(\theta(a)=0)$. In the case at hand, the world-sheet boundary curve $z(s)$ defines a set of zeros of $\hat{\theta}$, namely $\zeta_{s}$, for any $s$ is a zero of $\hat{\theta}$ (see eqs. (2.25), (2.26)). However, from the definition of $\hat{\theta}$ i.e. eq. (2.14) it is clear that $\hat{\theta}\left(\zeta_{s}\right)=0 \Longleftrightarrow \theta\left(\zeta_{s}+\int_{1}^{3}\right)=0$, it follows that we can take

$$
a=\zeta_{s}+\int_{1}^{3}
$$

Now choose the points $p_{i}=p_{4}, p_{k}=p_{3}, p_{l}=p_{1}, p_{j}=\overline{4}$. Remember that $p_{1}=0$ and $p_{3}=\infty$. Also $p_{4}$ and $p_{\overline{4}}$ are the same (arbitrary) point in the complex plane but on opposite sheets of the Riemann surface, implying $\int_{1}^{4}=-\int_{1}^{\overline{4}}$ since $p_{1}=0$ is a branch cut. Up to a constant independent of $\zeta_{s}$, the cross ratio can be written as

$$
\tilde{\gamma}=\frac{\theta\left(\zeta_{s}+\int_{1}^{4}\right)}{\theta\left(\zeta_{s}+\int_{1}^{\overline{4}}\right)} \frac{\hat{\theta}\left(\zeta_{s}+\int_{1}^{\overline{4}}\right)}{\hat{\theta}\left(\zeta_{s}+\int_{1}^{4}\right)}
$$

which is therefore independent of $\zeta_{s}$ as long as it is a zero of $\hat{\theta}$. However, it is also true that $\hat{\theta}(0)=0$. Replacing $\zeta_{s} \rightarrow 0$ and using that $\theta$ is symmetric whereas $\hat{\theta}$ is antisymmetric it immediately follows that $\tilde{\gamma}=-1$. Therefore the simple but very useful identity

$$
\frac{\theta\left(\zeta_{s}+\int_{1}^{4}\right)}{\theta\left(\zeta_{s}-\int_{1}^{4}\right)}=-\frac{\hat{\theta}\left(\zeta_{s}+\int_{1}^{4}\right)}{\hat{\theta}\left(\zeta_{s}-\int_{1}^{4}\right)} \quad \text { if } \quad \hat{\theta}\left(\zeta_{s}\right)=0
$$

is derived.

\section{A.4 First derivatives}

Identities for the derivatives of the theta functions can be obtained from the trisecant identity by deriving with respect to the position of the points $p_{i} p_{j}, p_{k}, p_{l}$. Consider deriving the trisecant identity with respect of the position of $p_{i}$ and afterwards taking the limit $p_{j} \rightarrow p_{i}$. The result reads

$$
D_{i} \ln \frac{\theta(\zeta)}{\theta\left(\zeta+\int_{k}^{l}\right)}=D_{i} \ln \frac{\hat{\theta}\left(\int_{l}^{i}\right)}{\hat{\theta}\left(\int_{k}^{i}\right)}-\frac{\hat{\theta}\left(\int_{l}^{k}\right) D_{i} \hat{\theta}(0)}{\hat{\theta}\left(\int_{i}^{k}\right) \hat{\theta}\left(\int_{l}^{i}\right)} \frac{\theta\left(\zeta+\int_{i}^{l}\right) \theta\left(\zeta+\int_{k}^{i}\right)}{\theta\left(\zeta+\int_{k}^{l}\right) \theta(\zeta)}
$$

valid for any vector $\zeta \in \mathbb{C}^{g}$ and any three pints $p_{i}, p_{k}, p_{l}$ in $\mathcal{M}$. In our case, such points are usually chosen among $p_{1}=0, p_{3}=\infty$, and $p_{4}, p_{\overline{4}}$ arbitrary and in opposite sheets of $\mathcal{M}$. As particular cases it is useful to derive

$$
\begin{aligned}
& D_{3} \ln \frac{\theta(\zeta)}{\theta\left(\zeta+\int_{1}^{4}\right)}=-D_{3} \ln \theta\left(\int_{1}^{4}\right)-\frac{\hat{\theta}\left(\int_{1}^{4}\right) D_{3} \hat{\theta}(0)}{\theta\left(\int_{1}^{4}\right) \theta(0)} \frac{\hat{\theta}(\zeta) \hat{\theta}\left(\zeta+\int_{1}^{4}\right)}{\theta(\zeta) \theta\left(\zeta+\int_{1}^{4}\right)} \\
& D_{3} \ln \frac{\hat{\theta}(\zeta)}{\hat{\theta}\left(\zeta+\int_{1}^{4}\right)}=-D_{3} \ln \theta\left(\int_{1}^{4}\right)+\frac{\hat{\theta}\left(\int_{1}^{4}\right) D_{3} \hat{\theta}(0)}{\theta\left(\int_{1}^{4}\right) \theta(0)} \frac{\theta(\zeta) \theta\left(\zeta+\int_{1}^{4}\right)}{\hat{\theta}(\zeta) \hat{\theta}\left(\zeta+\int_{1}^{4}\right)}
\end{aligned}
$$




$$
\begin{aligned}
& D_{1} \ln \frac{\theta(\zeta)}{\hat{\theta}\left(\zeta+\int_{1}^{4}\right)}=-D_{1} \ln \hat{\theta}\left(\int_{1}^{4}\right)+\frac{\theta\left(\int_{1}^{4}\right) D_{1} \hat{\theta}(0)}{\hat{\theta}\left(\int_{1}^{4}\right) \theta(0)} \frac{\hat{\theta}(\zeta) \theta\left(\zeta+\int_{1}^{4}\right)}{\theta(\zeta) \hat{\theta}\left(\zeta+\int_{1}^{4}\right)} \\
& D_{1} \ln \frac{\hat{\theta}(\zeta)}{\theta\left(\zeta+\int_{1}^{4}\right)}=-D_{1} \ln \hat{\theta}\left(\int_{1}^{4}\right)+\frac{\theta\left(\int_{1}^{4}\right) D_{1} \hat{\theta}(0)}{\hat{\theta}\left(\int_{1}^{4}\right) \theta(0)} \frac{\theta(\zeta) \hat{\theta}\left(\zeta+\int_{1}^{4}\right)}{\hat{\theta}(\zeta) \theta\left(\zeta+\int_{1}^{4}\right)}
\end{aligned}
$$

valid for any vector $\zeta$. The function $\hat{\theta}$ is defined in eq. (2.14) and is odd, a fact used in writing eq. (A.11). In the solutions eq. (2.22), the vector $\zeta$ is a function of the world-sheet coordinates $(z, \bar{z})$ :

$$
\zeta(z, \bar{z})=2 \omega_{1} \bar{z}+2 \omega_{3} z
$$

and therefore

$$
\partial_{z} F(\zeta(z, \bar{z}))=2 D_{1} F(\zeta), \quad \partial_{\bar{z}} F(\zeta(z, \bar{z}))=2 D_{3} F(\zeta)
$$

In that way, the identities (A.11) can be used as a table of derivatives of the theta functions and allow the verification of the equations of motion.

\section{A.5 Second derivatives}

Second derivatives can be computed in the same way as first ones. In this subsection we just derive a special case that is useful for our purposes. Consider eq. (A.10) and take the points $p_{i}=p_{3}, p_{l}=p_{4}$ and use the definition of $\hat{\theta}$, i.e. eq. (2.14). After reordering the terms, the result is

$$
D_{3} \ln \frac{\theta(\zeta)}{\hat{\theta}\left(\zeta+\int_{1}^{4}-\int_{3}^{k}\right)}=D_{3} \ln \frac{\hat{\theta}\left(\int_{3}^{k}\right)}{\theta\left(\int_{1}^{4}\right)}-\frac{D_{3} \hat{\theta}(0) \hat{\theta}\left(\zeta+\int_{1}^{4}\right)}{\theta\left(\int_{1}^{4}\right) \theta(\zeta)} \frac{\theta\left(\zeta-\int_{3}^{k}\right) \theta\left(\int_{1}^{4}-\int_{3}^{k}\right)}{\hat{\theta}\left(\int_{3}^{k}\right) \hat{\theta}\left(\zeta+\int_{1}^{4}-\int_{3}^{k}\right)}
$$

The pint $p_{k}$ is now taken to be very close to $p_{3}$ so that the expansion in eq. (2.16) can be used, namely

$$
\int_{3}^{k}=\omega_{3} \tilde{y}+\frac{1}{3} \tilde{y}^{3} \omega_{32}+\ldots
$$

with $\tilde{y}=\frac{2}{\sqrt{\lambda}}$. Multiplying eq. (A.14) by $\hat{\theta}\left(\int_{3}^{k}\right)$ and expanding in powers of $\tilde{y}$ gives, at order $\tilde{y}^{2}$,

$$
D_{3}^{2} \ln \left[\theta(\zeta) \theta\left(\int_{1}^{4}\right) \hat{\theta}\left(\zeta+\int_{1}^{4}\right)\right]+\left[D_{3} \ln \frac{\theta(\zeta) \theta\left(\int_{1}^{4}\right)}{\hat{\theta}\left(\zeta+\int_{1}^{4}\right)}\right]^{2}=\frac{D_{3}^{3} \hat{\theta}(0)}{D_{3} \hat{\theta}(0)}
$$

Similarly, taking $p_{i}=p_{1}$ and expanding fro $p_{k} \rightarrow p_{1}$ it follows that

$$
D_{1}^{2} \ln \left[\theta(\zeta) \hat{\theta}\left(\int_{1}^{4}\right) \theta\left(\zeta+\int_{1}^{4}\right)\right]+\left[D_{1} \ln \frac{\theta(\zeta) \hat{\theta}\left(\int_{1}^{4}\right)}{\theta\left(\zeta+\int_{1}^{4}\right)}\right]^{2}=\frac{D_{1}^{3} \hat{\theta}(0)}{D_{1} \hat{\theta}(0)}
$$

\section{A.6 First derivatives at $\zeta=\zeta_{s}$}

A special role in the construction is played by the world-sheet boundary curve $z(s)$ defined such that

$$
\hat{\theta}\left(\zeta_{s}\right)=0
$$


where

$$
\zeta_{s}=\zeta(z(s), \bar{z}(s))=2 \omega_{1} \bar{z}(s)+2 \omega_{3} z(s)
$$

It therefore becomes necessary to evaluate various derivatives at such values of $\zeta=\zeta_{s}$.

Consider the first and third equations in (A.11) and evaluate them at $\zeta=\zeta_{s}$. The result is

$$
\begin{aligned}
& D_{3} \ln \frac{\theta\left(\zeta_{s}\right)}{\theta\left(\zeta_{s}+\int_{1}^{4}\right)}=-D_{3} \ln \theta\left(\int_{1}^{4}\right) \\
& D_{1} \ln \frac{\theta\left(\zeta_{s}\right)}{\hat{\theta}\left(\zeta_{s}+\int_{1}^{4}\right)}=-D_{1} \ln \hat{\theta}\left(\int_{1}^{4}\right)
\end{aligned}
$$

Furthermore, using that $\hat{\theta}$ is antisymmetric and $\theta$ symmetric it follows that

$$
\begin{aligned}
& D_{3} \ln \frac{\theta\left(\zeta_{s}+\int_{1}^{4}\right)}{\theta\left(\zeta_{s}-\int_{1}^{4}\right)}=2 D_{3} \ln \theta\left(\int_{1}^{4}\right) \\
& D_{1} \ln \frac{\hat{\theta}\left(\zeta_{s}+\int_{1}^{4}\right)}{\hat{\theta}\left(\zeta_{s}-\int_{1}^{4}\right)}=2 D_{1} \ln \hat{\theta}\left(\int_{1}^{4}\right)
\end{aligned}
$$

Consider now the second equation in (A.11). Both sides have poles when $\hat{\theta}(\zeta)=0$ but after multiplying it by $\hat{\theta}(\zeta)$, it reads

$$
D_{3} \hat{\theta}(\zeta)+\hat{\theta}(\zeta) D_{3} \ln \frac{\theta\left(\int_{1}^{4}\right)}{\hat{\theta}\left(\zeta+\int_{1}^{4}\right)}=\frac{\hat{\theta}\left(\int_{1}^{4}\right) D_{3} \hat{\theta}(0)}{\theta\left(\int_{1}^{4}\right) \theta(0)} \frac{\theta(\zeta) \theta\left(\zeta+\int_{1}^{4}\right)}{\hat{\theta}\left(\zeta+\int_{1}^{4}\right)}
$$

Evaluating at $\zeta=\zeta_{s}$ gives

$$
D_{3} \hat{\theta}\left(\zeta_{s}\right)=\frac{\hat{\theta}\left(\int_{1}^{4}\right) D_{3} \hat{\theta}(0)}{\theta\left(\int_{1}^{4}\right) \theta(0)} \frac{\theta\left(\zeta_{s}\right) \theta\left(\zeta_{s}+\int_{1}^{4}\right)}{\hat{\theta}\left(\zeta_{s}+\int_{1}^{4}\right)}
$$

Similarly, from the last equation in (A.11) it follows that

$$
D_{1} \hat{\theta}\left(\zeta_{s}\right)=\frac{\theta\left(\int_{1}^{4}\right) D_{1} \hat{\theta}(0)}{\hat{\theta}\left(\int_{1}^{4}\right) \theta(0)} \frac{\theta\left(\zeta_{s}\right) \hat{\theta}\left(\zeta_{s}+\int_{1}^{4}\right)}{\theta\left(\zeta_{s}+\int_{1}^{4}\right)}
$$

Another possibility to cancel the pole at $\hat{\theta}(\zeta)=0$ is by subtracting the second equation in (A.11) from itself evaluated at $p_{\overline{4}}$ :

$$
D_{3} \ln \frac{\hat{\theta}\left(\zeta+\int_{1}^{4}\right)}{\hat{\theta}\left(\zeta-\int_{1}^{4}\right)}=2 D_{3} \ln \theta\left(\int_{1}^{4}\right)-\frac{\hat{\theta}\left(\int_{1}^{4}\right) D_{3} \hat{\theta}(0)}{\theta\left(\int_{1}^{4}\right) \theta(0)} \frac{\theta(\zeta)}{\hat{\theta}(\zeta)}\left[\frac{\theta\left(\zeta+\int_{1}^{4}\right)}{\hat{\theta}\left(\zeta+\int_{1}^{4}\right)}+\frac{\theta\left(\zeta-\int_{1}^{4}\right)}{\hat{\theta}\left(\zeta-\int_{1}^{4}\right)}\right]
$$

where again we used that $\hat{\theta}$ is odd: $\hat{\theta}\left(-\int_{1}^{4}\right)=-\hat{\theta}\left(\int_{1}^{4}\right)$. From the trisecant identity, taking the points $p_{i}=p_{4}, p_{j}=p_{1}, p_{k}=p_{3}, p_{l}=p_{\overline{4}}$ and using that $\int_{1}^{\overline{4}}=-\int_{1}^{4}$ one obtains

$$
\left[\frac{\theta\left(\zeta+\int_{1}^{4}\right)}{\hat{\theta}\left(\zeta+\int_{1}^{4}\right)}+\frac{\theta\left(\zeta-\int_{1}^{4}\right)}{\hat{\theta}\left(\zeta-\int_{1}^{4}\right)}\right]=\frac{\hat{\theta}\left(2 \int_{1}^{4}\right) \theta(0)}{\hat{\theta}\left(\int_{1}^{4}\right) \theta\left(\int_{1}^{4}\right)} \frac{\theta(\zeta) \hat{\theta}\left(\zeta+\int_{1}^{4}\right) \hat{\theta}\left(\zeta-\int_{1}^{4}\right)}{\hat{\theta}}
$$


Replacing in eq. (A.27), $\hat{\theta}(\zeta)$ indeed cancels resulting in

$$
D_{3} \ln \frac{\hat{\theta}\left(\zeta+\int_{1}^{4}\right)}{\hat{\theta}\left(\zeta-\int_{1}^{4}\right)}=2 D_{3} \ln \theta\left(\int_{1}^{4}\right)-\frac{\hat{\theta}\left(2 \int_{1}^{4}\right) D_{3} \hat{\theta}(0)}{\theta^{2}\left(\int_{1}^{4}\right)} \frac{\theta^{2}(\zeta)}{\hat{\theta}\left(\zeta+\int_{1}^{4}\right) \hat{\theta}\left(\zeta-\int_{1}^{4}\right)}
$$

which is now safe to evaluate at $\zeta=\zeta_{s}$. Similarly, from the last equation in eq. (A.11) it follows that

$$
D_{1} \ln \frac{\theta\left(\zeta+\int_{1}^{4}\right)}{\theta\left(\zeta-\int_{1}^{4}\right)}=2 D_{1} \ln \hat{\theta}\left(\int_{1}^{4}\right)-\frac{\theta\left(\int_{1}^{4}\right) D_{1} \hat{\theta}(0)}{\hat{\theta}\left(\int_{1}^{4}\right) \theta(0)} \frac{\theta(\zeta)}{\hat{\theta}(\zeta)}\left[\frac{\hat{\theta}\left(\zeta+\int_{1}^{4}\right)}{\theta\left(\zeta+\int_{1}^{4}\right)}+\frac{\hat{\theta}\left(\zeta-\int_{1}^{4}\right)}{\theta\left(\zeta-\int_{1}^{4}\right)}\right]
$$

leading to

$$
D_{1} \ln \frac{\theta\left(\zeta+\int_{1}^{4}\right)}{\theta\left(\zeta-\int_{1}^{4}\right)}=2 D_{1} \ln \hat{\theta}\left(\int_{1}^{4}\right)-\frac{\hat{\theta}\left(2 \int_{1}^{4}\right) D_{1} \hat{\theta}(0)}{\theta^{2}\left(\int_{1}^{4}\right)} \frac{\theta^{2}(\zeta)}{\theta\left(\zeta+\int_{1}^{4}\right) \theta\left(\zeta-\int_{1}^{4}\right)}
$$

\section{A.7 Higher derivatives at $\zeta=\zeta_{s}$}

Consider eq. (A.20). and take $p_{4} \rightarrow p_{3}$. Both sides develop a pole so we should carefully expand using eq. (2.16)

$$
\begin{aligned}
\omega\left(p_{4}\right) & =\omega_{3}+\tilde{y}^{2} \omega_{32}+\ldots \\
\int_{1}^{4} \omega & =\int_{1}^{3} \omega+\omega_{3} \tilde{y}+\frac{1}{3} \tilde{y}^{3} \omega_{32}+\ldots
\end{aligned}
$$

where $\tilde{y}=\frac{2}{\sqrt{\lambda}}$. It follows that

$$
\frac{D_{3} \hat{\theta}\left(\omega_{3} \tilde{y}+\frac{1}{3} \tilde{y}^{3} \omega_{32}+\ldots\right)}{\hat{\theta}\left(\omega_{3} \tilde{y}+\frac{1}{3} \tilde{y}^{3} \omega_{32}+\ldots\right)}=\frac{D_{3} \hat{\theta}\left(\zeta_{s}+\omega_{3} \tilde{y}+\frac{1}{3} \tilde{y}^{3} \omega_{32}+\ldots\right)}{\hat{\theta}\left(\zeta_{s}+\omega_{3} \tilde{y}+\frac{1}{3} \tilde{y}^{3} \omega_{32}+\ldots\right)}-D_{3} \ln \theta\left(\zeta_{s}\right)
$$

Expanding both sides in $\tilde{y}$ two useful identities are derived:

$$
\begin{aligned}
& \frac{D_{3}^{2} \hat{\theta}\left(\zeta_{s}\right)}{D_{3} \hat{\theta}\left(\zeta_{s}\right)}=2 D_{3} \ln \theta\left(\zeta_{s}\right) \\
& \frac{D_{3}^{3} \hat{\theta}\left(\zeta_{s}\right)}{D_{3} \hat{\theta}\left(\zeta_{s}\right)}=\frac{D_{3}^{3} \hat{\theta}(0)}{D_{3} \hat{\theta}(0)}-\frac{D_{3}^{\prime \prime} \hat{\theta}(0)}{D_{3} \hat{\theta}(0)}+\frac{D_{3}^{\prime \prime} \hat{\theta}\left(\zeta_{s}\right)}{D_{3} \hat{\theta}\left(\zeta_{s}\right)}+3\left(D_{3} \ln \theta\left(\zeta_{s}\right)\right)^{2}
\end{aligned}
$$

where $D_{3}^{\prime \prime}$ denotes the derivative along the direction $\omega_{32}$ and we also took into account that $\hat{\theta}$ is an odd function and therefore its even derivatives vanish at 0 . Similarly, starting from eq. (A.21) one finds

$$
\begin{aligned}
& \frac{D_{1}^{2} \hat{\theta}\left(\zeta_{s}\right)}{D_{1} \hat{\theta}\left(\zeta_{s}\right)}=2 D_{1} \ln \theta\left(\zeta_{s}\right) \\
& \frac{D_{1}^{3} \hat{\theta}\left(\zeta_{s}\right)}{D_{1} \hat{\theta}\left(\zeta_{s}\right)}=\frac{D_{1}^{3} \hat{\theta}(0)}{D_{1} \hat{\theta}(0)}-\frac{D_{1}^{\prime \prime} \hat{\theta}(0)}{D_{1} \hat{\theta}(0)}+\frac{D_{1}^{\prime \prime} \hat{\theta}\left(\zeta_{s}\right)}{D_{1} \hat{\theta}\left(\zeta_{s}\right)}+3\left(D_{1} \ln \theta\left(\zeta_{s}\right)\right)^{2}
\end{aligned}
$$


Finally, taking two derivatives in direction $\omega_{3}$ i.e. applying $D_{3}^{2}$ to eq. (A.24) and evaluating at $\zeta=\zeta_{s}$ follows that

$$
D_{3}^{2} \ln \left[\theta\left(\zeta_{s}\right) \theta\left(\zeta_{s}+\int_{1}^{4}\right) \hat{\theta}\left(\zeta_{s}+\int_{1}^{4}\right)\right]+\left(D_{3} \ln \frac{\theta\left(\zeta_{s}+\int_{1}^{4}\right)}{\hat{\theta}\left(\zeta_{s}+\int_{1}^{4}\right)}\right)^{2}=\frac{D_{3}^{3} \hat{\theta}\left(\zeta_{s}\right)}{D_{3} \hat{\theta}\left(\zeta_{s}\right)}-3\left(D_{3} \ln \theta\left(\zeta_{s}\right)\right)^{2}
$$

where eqs. (A.20), (A.25) and eq. (A.29) were used to simplify the result. Similarly

$D_{1}^{2} \ln \left[\theta\left(\zeta_{s}\right) \theta\left(\zeta_{s}+\int_{1}^{4}\right) \hat{\theta}\left(\zeta_{s}+\int_{1}^{4}\right)\right]+\left(D_{1} \ln \frac{\theta\left(\zeta_{s}+\int_{1}^{4}\right)}{\hat{\theta}\left(\zeta_{s}+\int_{1}^{4}\right)}\right)^{2}=\frac{D_{1}^{3} \hat{\theta}\left(\zeta_{s}\right)}{D_{1} \hat{\theta}\left(\zeta_{s}\right)}-3\left(D_{1} \ln \theta\left(\zeta_{s}\right)\right)^{2}$

is obtained. Instead for this derivation one can simply replace $\zeta=\zeta_{s}$ in eqs. (A.16), (A.17). The result is not exactly the same, they only agree if

$$
\frac{D_{3}^{3} \hat{\theta}\left(\zeta_{s}\right)}{D_{3}\left(\zeta_{s}\right)}-\frac{D_{3}^{2} \theta\left(\zeta_{s}\right)}{\theta\left(\zeta_{s}\right)}-2\left(D_{3} \ln \theta\left(\zeta_{s}\right)\right)^{2}=\frac{D_{3}^{3} \hat{\theta}(0)}{D_{3} \hat{\theta}(0)}-\frac{D_{3}^{2} \theta(0)}{\theta(0)}
$$

which is another useful identity.

\section{A.8 Other identities}

In [14] the following result was obtained

$$
\begin{aligned}
& \left(\frac{\theta(0) \hat{\theta}\left(\int_{1}^{4}\right)}{D_{1} \hat{\theta}(0) \theta\left(\int_{1}^{4}\right)}\right)^{2}=-4 \lambda_{p_{4}} \\
& \left(\frac{D_{3} \hat{\theta}(0) \hat{\theta}\left(\int_{1}^{4}\right)}{\theta(0) \theta\left(\int_{1}^{4}\right)}\right)^{2}=-\frac{1}{4} \lambda_{p_{4}}
\end{aligned}
$$

Consider now the first equation in eq. (A.11), apply $D_{3}$ and evaluate at $\zeta=\zeta_{s}$ :

$$
D_{3}^{2} \ln \frac{\theta\left(\zeta_{s}\right)}{\theta\left(\zeta_{s}+\int_{1}^{4}\right)}=-\left(\frac{\hat{\theta}\left(\int_{1}^{4}\right) D_{3} \hat{\theta}(0)}{\theta\left(\int_{1}^{4}\right) \theta(0)}\right)^{2}=\frac{1}{4} \lambda_{p_{4}}
$$

where eq. (A.25) and eq. (A.43) were used to simplify the result. Similarly, from the third equation in (A.11) and using eqs. (A.26), (A.42) it follows that

$$
D_{1}^{2} \ln \frac{\theta\left(\zeta_{s}\right)}{\hat{\theta}\left(\zeta_{s}+\int_{1}^{4}\right)}=\left(\frac{\theta\left(\int_{1}^{4}\right) D_{1} \hat{\theta}(0)}{\hat{\theta}\left(\int_{1}^{4}\right) \theta(0)}\right)^{2}=-\frac{1}{4 \lambda_{p_{4}}}
$$

These identities are valid whenever $\zeta_{s}$ is a zero of $\hat{\theta}$. In particular we can set $\zeta_{s}=0$ obtaining

$$
\begin{aligned}
& D_{1}^{2} \ln \hat{\theta}\left(\int_{1}^{4}\right)=\frac{1}{4 \lambda}+\frac{D_{1}^{2} \theta(0)}{\theta(0)} \\
& D_{3}^{2} \ln \theta\left(\int_{1}^{4}\right)=-\frac{\lambda}{4}+\frac{D_{3}^{2} \theta(0)}{\theta(0)}
\end{aligned}
$$


Open Access. This article is distributed under the terms of the Creative Commons Attribution License (CC-BY 4.0), which permits any use, distribution and reproduction in any medium, provided the original author(s) and source are credited.

\section{References}

[1] J.M. Maldacena, The large- $N$ limit of superconformal field theories and supergravity, Adv. Theor. Math. Phys. 2 (1998) 231 [Int. J. Theor. Phys. 38 (1999) 1113] [hep-th/9711200] [INSPIRE].

[2] S.S. Gubser, I.R. Klebanov and A.M. Polyakov, Gauge theory correlators from noncritical string theory, Phys. Lett. B 428 (1998) 105 [hep-th/9802109] [INSPIRE].

[3] E. Witten, Anti-de Sitter space and holography, Adv. Theor. Math. Phys. 2 (1998) 253 [hep-th/9802150] [INSPIRE].

[4] J.M. Maldacena, Wilson loops in large-N field theories, Phys. Rev. Lett. 80 (1998) 4859 [hep-th/9803002] [INSPIRE].

[5] S.-J. Rey and J.-T. Yee, Macroscopic strings as heavy quarks in large- $N$ gauge theory and anti-de Sitter supergravity, Eur. Phys. J. C 22 (2001) 379 [hep-th/9803001] [InSPIRE].

[6] N. Drukker, D.J. Gross and H. Ooguri, Wilson loops and minimal surfaces, Phys. Rev. D 60 (1999) 125006 [hep-th/9904191] [INSPIRE].

[7] D.E. Berenstein, R. Corrado, W. Fischler and J.M. Maldacena, The operator product expansion for Wilson loops and surfaces in the large-N limit, Phys. Rev. D 59 (1999) 105023 [hep-th/9809188] [INSPIRE].

[8] D.J. Gross and H. Ooguri, Aspects of large- $N$ gauge theory dynamics as seen by string theory, Phys. Rev. D 58 (1998) 106002 [hep-th/9805129] [INSPIRE].

[9] J.K. Erickson, G.W. Semenoff and K. Zarembo, Wilson loops in $N=4$ supersymmetric Yang-Mills theory, Nucl. Phys. B 582 (2000) 155 [hep-th/0003055] [INSPIRE].

[10] N. Drukker and D.J. Gross, An exact prediction of $N=4$ SUSYM theory for string theory, J. Math. Phys. 42 (2001) 2896 [hep-th/0010274] [INSPIRE].

[11] V. Pestun, Localization of gauge theory on a four-sphere and supersymmetric Wilson loops, Commun. Math. Phys. 313 (2012) 71 [arXiv:0712.2824] [InSPIRE].

[12] A.A. Tseytlin, Review of AdS/CFT integrability, chapter II.1: classical AdS $S_{5} \times S^{5}$ string solutions, Lett. Math. Phys. 99 (2012) 103 [arXiv:1012.3986] [INSPIRE].

[13] K. Pohlmeyer, Integrable Hamiltonian systems and interactions through quadratic constraints, Commun. Math. Phys. 46 (1976) 207 [INSPIRE].

[14] R. Ishizeki, M. Kruczenski and S. Ziama, Notes on Euclidean Wilson loops and Riemann Theta functions, Phys. Rev. D 85 (2012) 106004 [arXiv:1104.3567] [INSPIRE].

[15] M. Babich and A. Bobenko, Willmore Tori with umbilic lines and minimal surfaces in hyperbolic space, Duke Math. J. 72 (1993) 151 [INSPIRE].

[16] E.D. Belokolos, A.I. Bobenko, V.Z. Enol'skii, A.R. Its and V.B. Matveev, Algebro-geometric approach to nonlinear integrable equations, Springer-Verlag series in Non-linear Dynamics, Springer-Verlag, Berlin Heidelberg Germany and New York U.S.A. (1994).

[17] A. Jevicki and K. Jin, Moduli dynamics of AdS 3 strings, JHEP 06 (2009) 064 [arXiv:0903.3389] [INSPIRE]. 
[18] A. Jevicki, K. Jin, C. Kalousios and A. Volovich, Generating AdS string solutions, JHEP 03 (2008) 032 [arXiv:0712.1193] [INSPIRE].

[19] M. Kruczenski, Spiky strings and single trace operators in gauge theories, JHEP 08 (2005) 014 [hep-th/0410226] [INSPIRE].

[20] N. Dorey and B. Vicedo, On the dynamics of finite-gap solutions in classical string theory, JHEP 07 (2006) 014 [hep-th/0601194] [INSPIRE].

[21] K. Sakai and Y. Satoh, Constant mean curvature surfaces in AdS S , JHEP 03 (2010) 077 [arXiv: 1001.1553] [INSPIRE].

[22] H.J. De Vega and N.G. Sanchez, Exact integrability of strings in D-dimensional de Sitter space-time, Phys. Rev. D 47 (1993) 3394 [INSPIRE].

[23] A.L. Larsen and N.G. Sanchez, Sinh-Gordon, cosh-Gordon and Liouville equations for strings and multistrings in constant curvature space-times, Phys. Rev. D 54 (1996) 2801 [hep-th/9603049] [INSPIRE].

[24] N. Drukker, S. Giombi, R. Ricci and D. Trancanelli, Supersymmetric Wilson loops on $S^{3}$, JHEP 05 (2008) 017 [arXiv: 0711.3226] [INSPIRE].

[25] K. Zarembo, Wilson loop correlator in the AdS/CFT correspondence, Phys. Lett. B 459 (1999) 527 [hep-th/9904149] [INSPIRE].

[26] N. Drukker and B. Fiol, On the integrability of Wilson loops in $A d S_{5} \times S^{5}$ : some periodic ansätze, JHEP 01 (2006) 056 [hep-th/0506058] [INSPIRE].

[27] M. Kruczenski, A note on twist two operators in $N=4$ SYM and Wilson loops in Minkowski signature, JHEP 12 (2002) 024 [hep-th/0210115] [INSPIRE].

[28] L.F. Alday and J.M. Maldacena, Gluon scattering amplitudes at strong coupling, JHEP 06 (2007) 064 [arXiv: 0705. 0303] [INSPIRE].

[29] L.F. Alday and J. Maldacena, Null polygonal Wilson loops and minimal surfaces in anti-de-Sitter space, JHEP 11 (2009) 082 [arXiv:0904.0663] [INSPIRE].

[30] J. Maldacena and A. Zhiboedov, Form factors at strong coupling via a Y-system, JHEP 11 (2010) 104 [arXiv: 1009.1139] [INSPIRE].

[31] L.F. Alday, B. Eden, G.P. Korchemsky, J. Maldacena and E. Sokatchev, From correlation functions to Wilson loops, JHEP 09 (2011) 123 [arXiv: 1007.3243] [INSPIRE].

[32] L.F. Alday, D. Gaiotto, J. Maldacena, A. Sever and P. Vieira, An operator product expansion for polygonal null Wilson loops, JHEP 04 (2011) 088 [arXiv: 1006.2788] [INSPIRE].

[33] L.F. Alday, J. Maldacena, A. Sever and P. Vieira, Y-system for scattering amplitudes, J. Phys. A 43 (2010) 485401 [arXiv:1002.2459] [inSPIRE].

[34] B. Hoare and A.A. Tseytlin, Pohlmeyer reduction for superstrings in AdS space, J. Phys. A 46 (2013) 015401 [arXiv: 1209.2892] [InSPIRE].

[35] A. Irrgang and M. Kruczenski, Double-helix Wilson loops: case of two angular momenta, JHEP 12 (2009) 014 [arXiv: 0908.3020] [INSPIRE].

[36] M. Kruczenski, Spin chains and string theory, Phys. Rev. Lett. 93 (2004) 161602 [hep-th/0311203] [INSPIRE].

[37] B.A. Burrington and L.A. Pando Zayas, Phase transitions in Wilson loop correlator from integrability in global AdS, Int. J. Mod. Phys. A 27 (2012) 1250001 [arXiv:1012.1525] [INSPIRE]. 
[38] A. Dekel and T. Klose, Correlation function of circular Wilson loops at strong coupling, JHEP 11 (2013) 117 [arXiv: 1309.3203] [INSPIRE].

[39] R.A. Janik and P. Laskos-Grabowski, Surprises in the AdS algebraic curve constructions: Wilson loops and correlation functions, Nucl. Phys. B 861 (2012) 361 [arXiv:1203.4246] [INSPIRE].

[40] B. Fiol and G. Torrents, Exact results for Wilson loops in arbitrary representations, JHEP 01 (2014) 020 [arXiv: 1311.2058] [inSPIRE].

[41] D. Müller, H. Münkler, J. Plefka, J. Pollok and K. Zarembo, Yangian symmetry of smooth Wilson loops in $\mathcal{N}=4$ super Yang-Mills theory, JHEP 11 (2013) 081 [arXiv:1309.1676] [INSPIRE].

[42] S. Ryang, Algebraic curves for long folded and circular winding strings in $A d S_{5} \times S^{5}$, JHEP 02 (2013) 107 [arXiv: 1212.6109] [INSPIRE].

[43] A. Dekel, Algebraic curves for factorized string solutions, JHEP 04 (2013) 119 [arXiv:1302.0555] [INSPIRE].

[44] A. Irrgang and M. Kruczenski, Rotating Wilson loops and open strings in $A d S_{3}$, J. Phys. A 46 (2013) 075401 [arXiv: 1210.2298] [INSPIRE].

[45] V. Forini, V.G.M. Puletti and O. Ohlsson Sax, The generalized cusp in $A d S_{4} \times C P^{3}$ and more one-loop results from semiclassical strings, J. Phys. A 46 (2013) 115402 [arXiv: 1204.3302] [INSPIRE].

[46] G. Papathanasiou, Pohlmeyer reduction and Darboux transformations in Euclidean worldsheet $A d S_{3}$, JHEP 08 (2012) 105 [arXiv: 1203.3460] [INSPIRE].

[47] N. Drukker and V. Forini, Generalized quark-antiquark potential at weak and strong coupling, JHEP 06 (2011) 131 [arXiv: 1105.5144] [INSPIRE].

[48] B.A. Burrington, General Leznov-Savelev solutions for Pohlmeyer reduced AdS $S_{5}$ minimal surfaces, JHEP 09 (2011) 002 [arXiv:1105.3227] [INSPIRE].

[49] L.F. Alday and A.A. Tseytlin, On strong-coupling correlation functions of circular Wilson loops and local operators, J. Phys. A 44 (2011) 395401 [arXiv:1105.1537] [InSPIRE].

[50] C. Kalousios and D. Young, Dressed Wilson loops on $S^{2}$, Phys. Lett. B 702 (2011) 299 [arXiv:1104.3746] [INSPIRE].

[51] D. Mumford, Tata lectures in theta I \& II, with the collaboration of C. Musili, M. Nort, E. Previato and M. Stillman, Modern Birkhäuser Classics, Birkhäuser, Boston U.S.A. (2007).

[52] J.D. Fay, Theta functions on Riemann surfaces, Lectures Notes in Mathematics 352, Springer-Verlag, Berlin Heidelberg Germany and New York U.S.A. (1973).

[53] H.F. Baker, Abel's theorem and the allied theory, including the theory of the theta functions, Cambridge University Press, Cambridge U.K. (1897).

[54] H.M. Farkas and I. Kra, Riemann surfaces, Graduate Texts in Mathematics, Second Edition, Springer-Verlag, Berlin Heidelberg Germany and New York U.S.A. (1991).

[55] I.S. Gradshteyn and I.M. Ryzhik, Table of integrals series and products, sixth edition, Academic Press, San Diego U.S.A. and London U.K. (2000).

[56] O. Lechtenfeld, Superconformal ghost correlations on Riemann surfaces, Phys. Lett. B 232 (1989) 193 [INSPIRE]. 